\title{
A Numerical Study for Robust Active Portfolio Management with Worst-Case Downside Risk Measure
}

\author{
Aifan Ling ${ }^{1}$ and Le Tang ${ }^{2}$ \\ ${ }^{1}$ School of Finance, Jiangxi University of Finance \& Economics, Nanchang 330013, China
}

${ }^{2}$ Jiangxi University of Technology, Nanchang 330098, China

Correspondence should be addressed to Aifan Ling; aiffling@163.com

Received 9 December 2013; Accepted 22 January 2014; Published 1 April 2014

Academic Editor: Chuangxia Huang

Copyright (c) 2014 A. Ling and L. Tang. This is an open access article distributed under the Creative Commons Attribution License, which permits unrestricted use, distribution, and reproduction in any medium, provided the original work is properly cited.

Recently, active portfolio management problems are paid close attention by many researchers due to the explosion of fund industries. We consider a numerical study of a robust active portfolio selection model with downside risk and multiple weights constraints in this paper. We compare the numerical performance of solutions with the classical mean-variance tracking error model and the naive $1 / N$ portfolio strategy by real market data from China market and other markets. We find from the numerical results that the tested active models are more attractive and robust than the compared models.

\section{Introduction}

The choice of an optimal portfolio of assets has become a major research topic in financial economics. The meanvariance model proposed by Markowitz $(1952,1956)[1$, 2] provided a fundamental basis of portfolio selection for the theoretical and practical applications today. Analytical expression of the mean-variance efficient frontier could be derived by solving convex quadratic programs and the optimal portfolio can be found when the expected returns and covariance matrix of risk assets are exactly estimated. Based on Markowitz's mean-variance model, Roll (1992) [3] proposed an active portfolio management model which is called tracking error portfolio model in the literature. Roll [3] used the variance of tracking error to measure how closely a portfolio follows the index to which it is benchmarked. Motivated by Roll's seminal paper, many researches pay close attention to the active portfolio selection problems; see [4-9] and recent papers [10-18].

Let $\mathbf{r}=\left(r_{1}, \ldots, r_{n}\right)^{T} \in \mathbb{R}^{n}$ denote the return vector of the $n$ risk assets, where $r_{i}$ is the gross rate of return for the $i$ th risky asset. The investor's position is described by vector $\mathbf{w}=\left(w_{1}, \ldots, w_{n}\right)^{T} \in \mathbb{R}^{n}$, where the $i$ th component $w_{i}$ represents the proportion invested to the $i$ th risky asset. Let $\mathbf{w}_{b} \in \mathbb{R}^{n}$ denote a fixed portfolio that investor seeks to outperform. $\mathbf{w}_{b}$ also is called benchmark portfolio in the literature. Define the tracking error of portfolio $\mathbf{w}$ relative to benchmark portfolio $\mathbf{w}_{b}$ as $\Delta w=\left(\mathbf{w}-\mathbf{w}_{b}\right)^{T} \mathbf{r}$. In order to obtain a good tracking error portfolio $\mathbf{w}$, Roll [3] considered the solution of the following variance tracking error (VTE) problem

$$
\text { VTE: } \max \left\{\boldsymbol{\mu}^{T} \mathbf{w}:\left(\mathbf{w}-\mathbf{w}_{b}\right)^{T} \Sigma\left(\mathbf{w}-\mathbf{w}_{b}\right) \leq \sigma_{\text {vte }}, \sum_{i=1}^{n} w_{i}=1\right\},
$$

where $\boldsymbol{\mu}$ and $\Sigma$ are the expectation and covariance matrix of return vector $\mathbf{r}$, respectively, and $\sigma_{\mathrm{mv}}$ is the preset tracking error level. When $\boldsymbol{\mu}$ and $\Sigma$ are known exactly, similar to Markowitz's mean-variance portfolio, problem (1) can be solved as a convex quadratic programming.

Since the gain and the loss are symmetric on the mean value in the variance, Markowitz (1959) [19] proposed to use the semivariance of portfolio to control risk. Bawa (1975) [20], Bawa and Lindenberg (1977) [21], and Fishburn (1977) [22] later introduced a class of downside risk measure known as the lower partial moment (LPM) to better suit different risk profits of the investors. Because LPM mainly control the loss of portfolio, it becomes a popular risk controlling tool in theory and practice; see [23-28]. 
Generally, LPM can be expressed as

$$
\operatorname{LPM}_{m}(\rho)=\mathbb{E}\left[\left((\rho-X)_{+}\right)^{m}\right],
$$

where $X$ is the random variable, for example, the asset return of risky asset; $\rho$ may be a target that investors want to outperform, $m$ is a parameter, which can take any nonnegative value to model the risk attitude of an investor, $(a)_{+}=\max (a, 0)$, $\mathbb{E}[\cdot]$, and $\mathbb{P}\{\cdot\}$ below expresses the expectation and probability of random variable. For the case of $m=0,1,2$, we have

$$
\begin{aligned}
\operatorname{LPM}_{m}(\rho) & =\mathbb{E}\left[\left((\rho-X)_{+}\right)^{m}\right] \\
& = \begin{cases}\mathbb{P}\{X \leq \rho\}, & m=0 ; \\
\mathbb{E}\left[(\rho-X)_{+}\right], & m=1 ; \\
\mathbb{E}\left[\left((\rho-X)_{+}\right)^{2}\right], & m=2 .\end{cases}
\end{aligned}
$$

That is to say that $\mathrm{LPM}_{0}$ is nothing but the probability of the asset return falling below the benchmark index $\mathrm{LPM}_{1}$ is the expected shortfall of the investment falling below the benchmark index and $\mathrm{LPM}_{2}$ is an analog of the semivariance; here, however, the deviation is in reference to a preset target or benchmark return instead of the mean.

In the last five to ten years, robust portfolio optimization problems based on the robust optimization technique developed by Ben-Tal and Nemirovski (1998) [29] are the focus of many financial and economic researchers. Based on robust portfolio optimization theory, one can deal with models with uncertainty parameters or uncertainty distribution. For instance, Costa and Paiva (2002) [30] considered a robust framework of model (1) with $\boldsymbol{\mu}$ and $\Sigma$ in a polytopic uncertainty set described by its vertices. El Ghaoui et al. (2003) [31] proposed a worst-case Value-atRisk (VaR) robust optimization model in which they assumed that the distribution of returns is partially known. Only bounds on the mean and covariance matrix are available in El Ghaoui et al's model and the proposed model can be solved by a second order cone programming approach. Goldfarb and Iyengar (2003) [32] proposed a robust factor model and later their models were developed and extended by Erdoğan et al. (2008) [33] to a robust index tracking and active portfolio management problem; see also Ling and $\mathrm{Xu}$ (2012) [34] for a similar research. Zhu et al. (2009) [28] proposed a robust framework based on LPM constraints with uncertainty discrete distribution. Glabadanidis (2010) [11] considered a robust and efficient strategy to track and outperform a benchmark in which he proposed a sequential stepwise regression and relative method based on factor models of security returns. Chen et al. (2011) [23] developed some tight bounds on the expected values of LPM under the framework of robust optimization models arising from portfolio selection problems. More robust portfolio models based on different parameters uncertainty or distribution uncertainty can be found in the recent survey given by Fabozzi et al. (2010) [35].

As we know, some policies are commonly found in the contracts between investors and portfolio managers and require that the weights of certain types of assets should be smaller, higher, or equal to a given percentage in some funds. We call this type of restriction in this paper weights constraint(s) of a portfolio. For instance, weights constraint appears frequently in some index funds (ETF), stock style funds (the weights invested in stocks is not less than a preset percentage of the market value of fund), bond style funds (the weights invested in stocks is not larger than a preset value), QFII (Qualified foreign institutional investors) funds, and QDII (Qualified domestic institutional investor) funds require that the weights invested in foreign markets not be larger than a preset value. However, it is to be regretted that weights constraint is rarely used in the current tracking error (robust) portfolio literature. Recently, Bajeux-Besnainou et al. (2011) [36] first introduce this kind of constraint into a mean-variance index tracking portfolio model. In their paper, Bajeux-Besnainou et al. described a comparison with and without weights constraint and showed that the influence of weights constraint is very remarkable and cannot be ignored for the returns of portfolio.

Motivated by the topics of active portfolio management that are paid close attention by many researchers and the fact that there exists the rare model with explicit solutions in the robust active portfolio management literature, in this paper, we further consider a numerical study of robust active portfolio selection model that is proposed by Ling et al. in [37]. In the current framework, we test the models in [37] using the real market data which includes ten stock indexes from Shanghai Stock Exchange (SHH) and Shenzhen Stock Exchange (SHZ), which are called domestic assets, and four stock indexes from other stock exchanges which are called foreign assets. Our numerical studies give the comparisons with the classical (VTE) and the naive $1 / N$ portfolio strategy considered by DeMiguel et al. (2009) [38]. Numerical results indicate that the proposed active portfolio selection model can obtain better numerical performance for real market data. Most of proofs of theorems in the current paper can be found in [37], but, in order to keep the completeness of reading, we still give these main proofs in Appendix.

This paper is arranged as follows. In Section 2, we give the robust active portfolio problem with multiple weights constraints and establish the robust active portfolio models. We explore the explicit solutions of the proposed models in Section 3. In Section 4, we do some numerical tests and comparisons based on real market data.

\section{Robust Active Portfolio Problems}

Let $\mathscr{I}$ be a subset of $\{1,2, \ldots, n\}$. If assets in $\mathscr{I}$ are restricted to a limited weight, we call $\mathscr{I}$ the set of restricted assets. For given constant $q$, weights constraint requires

$$
\mathbf{e}_{\mathscr{g}}^{T} \mathbf{w}=(\leq) q
$$

where $\mathbf{e}_{\mathscr{I}}=\left(e_{1}, \ldots, e_{n}\right)^{T} \in \mathbb{R}^{n}$ is an index vector with $e_{i}=1$ when $i \in \mathscr{I}$, and $e_{i}=0$ when $i \notin \mathscr{I}$. The notation " $=(\leq$ )" means the equality only (or inequality only) weights constraint. Under the estimation of $\boldsymbol{\mu}$ and $\Sigma$ is exactly obtained 
and selling short is allowed, Bajeux-Besnainou et al. [36] considered the following active portfolio optimization problem with single weight constraint:

$$
\begin{gathered}
\max \left\{\mathbb{E}\left[\mathbf{w}^{T} \mathbf{r}\right]:\left(\mathbf{w}-\mathbf{w}_{b}\right)^{T} \sum\left(\mathbf{w}-\mathbf{w}_{b}\right) \leq \sigma,\right. \\
\left.\mathbf{e}_{\mathscr{J}}^{T} \mathbf{w}=(\leq) q, \mathbf{e}^{T} \mathbf{w}=1\right\}
\end{gathered}
$$

and get a closed solution of this problem. As pointed in Section 1, the classical mean-variance framework may not be appropriate since it controls not only the loss of portfolio, but also the gain of portfolio.

In our framework, we use LPM to control the risk of portfolio by which we in fact only control the loss of portfolio. Additionally, we make no assumption on the distribution of returns r. Instead, we assume that we only partially know some knowledge about the statistical properties of returns $\mathbf{r}$ and the underlying distribution function is only known to belong to a certain set $\mathscr{D}$ of distribution functions $\mathscr{F}$ of the returns vector $\mathbf{r}$. Under the uncertainty set $\mathscr{D}$ of the underlying distribution, we have the worst-case $\mathrm{LPM}_{m}$.

Definition 1 . For any $m \geq 0$ and real number $\rho$, the worstcase lower-partial moment (WCLPM for short) of random variable $X$ with respect to $\rho$ under $\mathscr{F} \in \mathscr{D}$ is defined by

$$
\mathrm{WCLPM}_{m}(\rho)=\sup _{\mathscr{F} \in \mathscr{D}} \mathbb{E}\left[\left((\rho-X)_{+}\right)^{m}\right] .
$$

In tracking error portfolio selection problems, investors want in fact to find a portfolio $\mathbf{w}$, such that the return of portfolio $\mathbf{w}$ can be close to or outperform the return of benchmark $\mathbf{w}_{b}^{T} \boldsymbol{\mu}$. For this, we call $\mathbf{w}_{b}^{T} \boldsymbol{\mu}-\mathbf{w}^{T} \boldsymbol{\mu}$ the loss of portfolio $\mathbf{w}$ relative to $\mathbf{w}_{b}^{T} \boldsymbol{\mu}$ and control the risk of portfolio by restricting the loss. Based on this idea, we maximize on one hand the return $\mathbf{w}^{T} \boldsymbol{\mu}$ and on the other hand control the loss by using worst-case $\mathrm{LPM}_{m}$. Thus, the mean-WCLPM ${ }_{m}$ robust tracking error problem with multiple weights constraints can be written as

$$
\begin{array}{ll}
\max _{\mathbf{w}} & \mathbf{w}^{T} \boldsymbol{\mu} \\
\text { s.t. } & \sup \mathbb{E}\left[\left(\left(\mathbf{w}_{b}^{T} \mathbf{r}-\mathbf{w}^{T} \mathbf{r}\right)_{+}\right)^{m}\right] \leq \sigma_{m} \\
& \mathbb{F}_{\mathscr{F}}^{T} \mathbf{e}_{\mathscr{g}}^{T} \mathbf{w}=(\leq) q_{i}, \quad i=1, \ldots, p, \\
& \mathbf{e}^{T} \mathbf{w}=1,
\end{array}
$$

where $\sigma_{m}(m=0,1,2)$ are the preset constants. The second class of inequalities constraints; $p$ weights constraints express that several classes of assets are restricted to the limited weights, where $\mathscr{I}_{i} \subseteq\{1,2, \ldots, n\}$ and $q_{i}(i=1, \ldots, p)$ are constants. We call these constraints multiple weights constraints. When $p=1$, it is the single weight constraint. In this paper, we only consider the cases of $m=0,1,2$ and multiple equality weights constraints. The corresponding results with multiple inequality weights constraints can be obtained by the similar methods.

Because we are interested in developing robust tracking error portfolio policies and exploring the explicit solutions of problems (7), in the rest of this paper, we assume that $\mathscr{D}$ is the set of allowable distributions of returns $\mathbf{r}$ with the known mean and covariance; that is,

$$
\mathscr{D}=\{\mathbf{r} \mid \mathbb{E}[\mathbf{r}]=\boldsymbol{\mu}, \operatorname{Cov}(\mathbf{r})=\Sigma>0\} .
$$

For convenience, we sometimes denote $\mathbf{r} \in \mathscr{D}$ by $\mathbf{r} \sim(\boldsymbol{\mu}, \Sigma)$. Our model can be extended to the case of parameters uncertainty, for which parameters are not estimated exactly.

Similar to Erdoğan et al. [33] and Bajeux-Besnainou et al. [36], we normalize the benchmark portfolio $\mathbf{w}_{b}$; that is, $\mathbf{e}^{T} \mathbf{w}_{b}=1$ and introduce a new variable $\mathbf{y}=\mathbf{w}-\mathbf{w}_{b}$. Notice the $\mathbf{w}^{T} \boldsymbol{\mu}=\mathbf{y}^{T} \boldsymbol{\mu}+\mathbf{w}_{b}^{T} \boldsymbol{\mu}$; then optimization problem (7) with multiple equality weights constraints can be written into the following form:

$$
\begin{aligned}
\text { (RSm): } \max _{\mathbf{y}} & \mathbf{y}^{T} \boldsymbol{\mu} \\
\text { s.t. } & \sup _{\mathbf{r} \in \mathscr{D}} \mathbb{E}\left[\left(\left(-\mathbf{y}^{T} \mathbf{r}\right)_{+}\right)^{m}\right] \leq \sigma_{m} \\
& \mathbf{e}_{\mathscr{G}_{i}}^{T} \mathbf{y}=q_{i}-\mathbf{e}_{\mathscr{F}_{i}}^{T} \mathbf{w}_{b}, \quad i=1, \ldots, p, \\
& \mathbf{e}^{T} \mathbf{y}=0 .
\end{aligned}
$$

We call $\mathbf{y}$ a self-financing portfolio and $\mathbf{w}$ a fully investing portfolio. We mention that $q_{i}-\mathbf{e}_{\mathscr{F}_{i}}^{T} \mathbf{w}_{b}, i=1, \ldots, p$ can be positive or negative, most likely between -1 and 1 since $q_{i}$ and $\mathbf{e}_{\mathscr{F}_{i}}^{T} \mathbf{w}_{b}$ are in most cases between 0 and 1 . In the rest of the paper, we suppose that $p+1$ vectors $\mathbf{e}$ and $\mathbf{e}_{\mathscr{I}_{i}}(i=1, \ldots, p)$ are linearly independent and $n>p+1$ always holds. If there exists certain $\mathbf{e}_{\mathscr{I}_{k}}$, such that $\mathbf{e}_{\mathscr{I}_{k}}$ can be expressed as the linear combination of $\mathbf{e}_{\mathscr{I}_{i}}(i=1, \ldots, p, i \neq k)$, then $\mathbf{e}_{\mathscr{F}_{k}}^{T} \mathbf{y}=$ $q_{k}-\mathbf{e}_{\mathscr{J}_{k}}^{T} \mathbf{w}_{b}$ becomes a redundant constraint. Additionally, the linear equality constraints will lead to a unique feasible solution $\mathrm{y}$ if $n=p+1$.

The following lemmas indicate that a tight upper bound of the worst-case $\mathrm{LPM}_{m}$ can be obtained explicitly, which will be helpful for our analysis later. We extend the proof in [23] for $\mathrm{WCLPM}_{0}$ to a general case.

Lemma 2 (see $[23,39])$. Let $\xi$ be a random variable with mean $\mu$ and variance $\sigma$ but have unknown distribution and $\rho$ is an arbitrary real number. Then, we have the following.

(a) For the case of $m=0$,

$$
\sup _{\xi \sim(\mu, \sigma)} \mathbb{P}\{\xi \leq \rho\}= \begin{cases}\frac{1}{1+(\rho-\mu)^{2} / \sigma^{2}}, & \text { if } \rho \leq \mu ; \\ 1, & \text { if } \rho \geq \mu .\end{cases}
$$

(b) For the case of $m=1$,

$$
\sup _{\xi \sim(\mu, \sigma)} \mathbb{E}\left[(\rho-\xi)_{+}\right]=\frac{\rho-\mu+\sqrt{\sigma^{2}+(\rho-\mu)^{2}}}{2} .
$$

(c) For the case of $m=2$,

$$
\begin{aligned}
\sup _{\xi \sim(\mu, \sigma)} \mathbb{E}\left[\left((\rho-\xi)_{+}\right)^{2}\right] & =\left[(\rho-\mu)_{+}\right]^{2}+\sigma^{2} \\
& = \begin{cases}\sigma^{2}, & \rho \leq \mu ; \\
(\rho-\xi)^{2}+\sigma^{2}, & \rho \geq \mu .\end{cases}
\end{aligned}
$$


Lemma 3 (see $[40]$ ). For any $\mathbf{a} \in \mathbb{R}^{n}$, denote

$$
\begin{gathered}
S_{1}=\left\{\mathbf{a}^{T} \mathbf{r} \mid \mathbf{r} \in \mathscr{D}\right\}, \\
S_{2}=\left\{\eta \mid \mathbb{E}(\eta)=\mathbf{a}^{T} \boldsymbol{\mu}, \operatorname{Var}(\eta)=\mathbf{a}^{T} \sum \mathbf{a}\right\} .
\end{gathered}
$$

Then $S_{1}=S_{2}$.

Lemma 4 (see [37]). For any $\mathbf{r} \in \mathscr{D}, \eta \in S_{2}$, and any real vector $\mathbf{a} \in \mathbb{R}^{n}$, the following equality

$$
\sup _{\mathbf{r} \in \mathscr{D}} \mathbb{E}\left[\left(\left(\rho-\mathbf{a}^{T} \mathbf{r}\right)_{+}\right)^{m}\right]=\sup _{\eta \sim\left(\mathbf{a}^{T} \boldsymbol{\mu}, \mathbf{a}^{T} \Sigma \mathbf{a}\right)} \mathbb{E}\left[\left((\rho-\eta)_{+}\right)^{m}\right]
$$

holds.

Lemma 4 establishes the relationship of single variable $\eta$ and multivariable $\mathbf{r}$, which is useful for our analysis later. We end this section by introducing some notations that will be used frequently. Unless otherwise specified, in the rest of paper, bold letter expresses a vector, uppercase letter is a matrix, and lowercase is a real number. $\mathbf{0}$ is a zero matrix or zero vector by context. For simplicity, we denote $\mathbf{e}_{\mathscr{I}_{k}}$ by $\mathbf{e}_{k}$, $k=1, \ldots, p$ and $q_{i}-\mathbf{e}_{\mathscr{F}_{i}}^{T} \mathbf{w}_{b}$ by $d_{i}$ and thus $d_{i} \in[-1,1]$. Let

$$
M=\left[\mathbf{e}_{1}, \ldots, \mathbf{e}_{p}\right] \in \mathbb{R}^{n \times p}, \quad \mathbf{d}=\left(d_{1}, \ldots, d_{p}\right)^{T} \in \mathbb{R}^{p} .
$$

Then, from the assumption that $\mathbf{e}_{1}, \ldots, \mathbf{e}_{p}$ and $\mathbf{e}$ are linearly independent, matrix $M$ has column full rank. Let $A=$ $M^{T} \Sigma^{-1} M \in \mathbb{R}^{p \times p}$. Then matrix $A$ is positive definite via $\Sigma^{-1}>0$, where the notation $Z \succeq Y$ (or $Z>Y$ ) means matrix $Z-Y$ is positive semidefinite (or positive definite). Further, we denote

$$
B=I-M A^{-1} M^{T} \Sigma^{-1} \in \mathbb{R}^{n \times n}, \quad C=\Sigma^{-1} M A^{-1} \in \mathbb{R}^{n \times p},
$$

where $I$ is a unit matrix with reasonable dimensions. Then, for these matrices, we have the following results.

Lemma 5. (a) $B M=\mathbf{0}$.
(b) $M^{T} \Sigma^{-1} B=\mathbf{0}$
(c) $B^{T} C=\mathbf{0}$.
(d) $B^{T} \Sigma^{-1} B=\Sigma^{-1} B$
(e) $C^{T} \sum C=A^{-1}$.
(f) Matrix $B$ is positive semidefinite.

The conclusions are straightforward for (a)-(e). For (f), we have from (d), $\Sigma^{-1} B=B^{T} \Sigma^{-1} B \succeq \mathbf{0}$ since $\Sigma^{-1}>\mathbf{0}$. Hence $B$ is a positive semidefinite matrix.

Based on matrices $B, C$, and $\Sigma^{-1}$, we denote additionally

$$
\begin{array}{rrr}
a=\mathbf{e}^{T} \Sigma^{-1} \mathbf{e}, & b=\mathbf{e}^{T} \Sigma^{-1} \boldsymbol{\mu}, & c=\boldsymbol{\mu}^{T} \Sigma^{-1} \boldsymbol{\mu}, \\
a_{0}=\mathbf{e}^{T} C \mathbf{d}, & b_{0}=\boldsymbol{\mu}^{T} C \mathbf{d}, & c_{0}=\mathbf{d}^{T} A^{-1} \mathbf{d}, \\
a_{1}=\mathbf{e}^{T} \Sigma^{-1} B \mathbf{e}, & b_{1}=\mathbf{e}^{T} \Sigma^{-1} B \boldsymbol{\mu}, & c_{1}=\boldsymbol{\mu}^{T} \Sigma^{-1} B \boldsymbol{\mu}, \\
a_{2}=a_{1} c_{1}-b_{1}^{2}, & b_{2}=a_{1} b_{0}-a_{0} b_{1}, & c_{2}=a_{1} c_{0}+a_{0}^{2} .
\end{array}
$$

Without loss the generality, we assume $\Sigma^{-1} B \neq \mathbf{0}$; then we have that

$$
\begin{gathered}
a c>b^{2}, \quad a_{1}>0, \quad c_{1}>0, \\
a_{2}>0, \quad c_{2} \geq 0,
\end{gathered}
$$

and $c_{2}=0$ if and only if $\mathbf{d}=\mathbf{0}$ via $A^{-1}>\mathbf{0}$.

\section{The Solutions}

In this section, we will explore the explicit solution of problem (RSm). Notice that $\mathbf{y}=\mathbf{w}-\mathbf{w}_{b}$; then we can rewrite separably problem (RSm) into the following three problems:

$$
\begin{aligned}
& \max _{\mathbf{y}}\left\{\mathbf{y}^{T} \boldsymbol{\mu}: \sup _{\mathbf{r} \in \mathscr{D}} \mathbb{P}\left\{\mathbf{y}^{T} \mathbf{r} \leq 0\right\} \leq \sigma_{0}, M^{T} \mathbf{y}=\mathbf{d}, \mathbf{e}^{T} \mathbf{y}=0\right\}, \\
& \max _{\mathbf{y}}\left\{\mathbf{y}^{T} \boldsymbol{\mu}: \sup _{\mathbf{r} \in \mathscr{D}} \mathbb{E}\left[\left(-\mathbf{y}^{T} \mathbf{r}\right)_{+}\right] \leq \sigma_{1}, M^{T} \mathbf{y}=\mathbf{d}, \mathbf{e}^{T} \mathbf{y}=0\right\},
\end{aligned}
$$

$$
\max _{\mathbf{y}}\left\{\mathbf{y}^{T} \boldsymbol{\mu}: \sup _{\mathbf{r} \in \mathscr{D}} \mathbb{E}\left[\left(\left(-\mathbf{y}^{T} \mathbf{r}\right)_{+}\right)^{2}\right] \leq \sigma_{2}, M^{T} \mathbf{y}=\mathbf{d}, \mathbf{e}^{T} \mathbf{y}=0\right\}
$$

which is corresponding to the case of $\rho=0$ in the worst-case $\mathrm{LPM}_{m}$.

3.1. Maximization of Information Ratio: A Variation of $W C L P M_{0}$. Let us first consider problem (19). The inequality $\mathbb{P}\left\{\mathbf{y}^{T} \mathbf{r} \leq 0\right\} \leq \sigma_{0}$ is usually called chance constraint or Roy's safety-first rule in the literature. Generally speaking, $\sigma_{0}$ is taken far less than $1 / 2$ which leads to $\left(1 / \sigma_{0}\right)-1>0$. Hence, from Lemma 2(a) and Lemma 4, we have that, when $\mathbf{y}^{T} \boldsymbol{\mu} \geq 0$,

$$
\begin{aligned}
\sup _{\mathbf{r} \in \mathscr{D}} \mathbb{P}\left\{\mathbf{y}^{T} \mathbf{r} \leq 0\right\} \leq \sigma_{0} & \Longleftrightarrow \frac{1}{1+\left(-\mathbf{y}^{T} \boldsymbol{\mu}\right)^{2} / \mathbf{y}^{T} \Sigma \mathbf{y}} \leq \sigma_{0} \\
& \Longleftrightarrow \frac{\left(-\mathbf{y}^{T} \boldsymbol{\mu}\right)^{2}}{\mathbf{y}^{T} \Sigma \mathbf{y}} \geq\left(\frac{1}{\sigma_{0}}-1\right) \\
& \Longleftrightarrow \frac{\mathbf{y}^{T} \boldsymbol{\mu}}{\sqrt{\mathbf{y}^{T} \Sigma \mathbf{y}}} \geq \sqrt{\left(\frac{1}{\sigma_{0}}-1\right) .}
\end{aligned}
$$

The last inequality means that information ratio (or sharpe ratio) of the portfolio is not less than a preset constant. By this characteristic, we consider a variation version of problem (19), in which we minimize the WCLPM $\mathrm{W}_{0}$; that is,

$$
\min _{\mathbf{y}}\left\{\sup _{\mathbf{r} \in \mathscr{D}} \mathbb{P}\left\{\mathbf{y}^{T} \mathbf{r} \leq 0\right\} \mid \text { s.t. } M^{T} \mathbf{y}=\mathbf{d}, \mathbf{e}^{T} \mathbf{y}=0\right\},
$$

which results from (22) the maximization of information ratio (IR for short):

$$
\operatorname{MAX}_{\mathrm{IR}}=\max _{\mathbf{y}}\left\{\frac{\mathbf{y}^{T} \boldsymbol{\mu}}{\sqrt{\mathbf{y}^{T} \Sigma \mathbf{y}}}: M^{T} \mathbf{y}=\mathbf{d}, \mathbf{e}^{T} \mathbf{y}=0\right\} \text {. }
$$


Problem (24) is related to the mean-variance with multiple weights constraints (MVMWC, for short) for a given parameter $\sigma_{0}^{\prime}$ :

$$
\text { (MVMWC): } \max _{\mathbf{y}}\left\{\mathbf{y}^{T} \boldsymbol{\mu}: \mathbf{y}^{T} \Sigma \mathbf{y} \leq \sigma_{0}^{\prime}, M^{T} \mathbf{y}=\mathbf{d}, \mathbf{e}^{T} \mathbf{y}=0\right\} .
$$

If $p=0$, that is, there is not weights constraint, then problem (25) is nothing but Roll's VTE problem. If $p=1$, that is, $M=\mathbf{e}_{1}$, then problem (25) becomes the case of single weight constraint problem which has been considered by BajeuxBesnainou et al. [36].

The explicit solution of problem (24) without weights constraints has been obtained in the literature (e.g., see [16, 23] for detail). But the methods in the literature cannot be used directly for problem (24) because of the existence of multiple weights constraints. Thus, we need to find the different method from the literature to obtain the explicit solution. To this end, we deal with this problem in two stages to get the explicit solution of (24). We introduce a parameter at first stage and parameterize the solution of (24), and then maximize IR of the solution with respect to the parameter in the second stage.

Theorem 6. There exists a real number, say $\tau_{0}$, such that the explicit solution of problem (24) can be expressed as

$$
\mathbf{y}_{\tau_{0}}^{*}=\frac{1}{\tau_{0}}\left(\Sigma^{-1} B \boldsymbol{\mu}-\frac{b_{1}}{a_{1}} \Sigma^{-1} B \mathbf{e}\right)+a_{0}\left(\frac{C \mathbf{d}}{a_{0}}-\frac{\Sigma^{-1} B \mathbf{e}}{a_{1}}\right) .
$$

Proof. See Theorem 1 in [37]; see also Appendix for detail.

To obtain the portfolio with the maximum information ratio, that is, the optimal solution of problem (24), what is then the value of parameter $\tau_{0}$ ? As the second stage, we replace $\mathbf{y}_{\tau_{0}}^{*}$ into the objective function (24). Then we get the information ratio of portfolio $\mathbf{y}_{\tau_{0}}^{*}$, denoted by $\mathrm{MAX}_{\tau_{0}}$, which is a function of parameter $\tau_{0}$. Thus, we can get the maximum information ratio by maximizing $\mathrm{MAX}_{\tau_{0}}$ with respect to $\tau_{0}$. That is, we have

$$
\operatorname{MAX}_{\mathrm{IR}}=\max _{\tau_{0}>0} \operatorname{MAX}_{\tau_{0}}=\max _{\tau_{0}>0} \frac{\left(\mathbf{y}_{\tau_{0}}^{*}\right)^{T} \boldsymbol{\mu}}{\sqrt{\left(\mathbf{y}_{\tau_{0}}^{*}\right)^{T} \Sigma \mathbf{y}_{\tau_{0}}^{*}}} .
$$

Let $\tau_{0}^{*}$ be the optimal solution of (27); then portfolio $\mathbf{y}_{\tau_{0}^{*}}^{*}$ is the optimal solution of (24), for which it has the maximum information ratio. Hence, we have the results below and its proof is straightforward.

Theorem 7. The maximizer of problem (27) is given by

$$
\tau_{0}^{*}=\frac{a_{1} b_{0}-a_{0} b_{1}}{a_{1} c_{0}+a_{0}^{2}}
$$

when $a_{1} b_{0}-a_{0} b_{1}>0$ and the optimal solution of (24) is obtained by

$$
\begin{aligned}
\mathbf{y}_{\mathrm{IR}}^{*}= & \frac{\left(a_{1} c_{0}+a_{0}^{2}\right)}{a_{1} b_{0}-a_{0} b_{1}}\left(\Sigma^{-1} B \boldsymbol{\mu}-\frac{b_{1}}{a_{1}} \Sigma^{-1} B \mathbf{e}\right) \\
& +a_{0}\left(\frac{C \mathbf{d}}{a_{0}}-\frac{\Sigma^{-1} B \mathbf{e}}{a_{1}}\right) .
\end{aligned}
$$

Theorem 8 (see [37]). If $\mathbf{y}_{\tau_{0}}^{*}$ in (26) is obtained for certain positive number $\tau_{0}$, then, there exists a $\sigma_{0}^{\prime}>0$, such that both problems (24) and (25) have the same optimal solution.

Let $\mathbf{y}_{0}^{*}$ be the optimal solution of (24); in view of the proof of Theorem 6 in Appendix, if $\sigma_{0}^{\prime}$ is chosen and satisfies

$$
\frac{\sqrt{a_{2}\left(a_{1} \sigma_{0}^{\prime}-c_{2}\right)}}{a_{2}}=\frac{\left(\mathbf{y}_{0}^{*}\right)^{T} \Sigma \mathbf{y}_{0}^{*}}{\left(\boldsymbol{\mu}^{T} \mathbf{y}_{0}^{*}\right)},
$$

then the optimal portfolio for problem (25) is also the optimal solution of (24). The relationship between problems (24) and (25) can be presented as follows. Solving (24) will get a portfolio with the maximum IR. However, the optimal portfolio of problem (25) is not necessarily the one with the maximum IR. If we solve (25) with $\sigma_{0}^{\prime}$ given by (30), then the optimal portfolio of (25) will have the maximum IR, too.

3.2. The Mean-WCLPM $M_{1}$. The WCLPM $\mathrm{W}_{1}$ constraint in problem (20) from Lemmas 2 and 4 can be written as

$$
\begin{aligned}
\sup _{\mathbf{r} \in \mathscr{D}} \mathbb{E}\left[\left(-\mathbf{y}^{T} \mathbf{r}\right)_{+}\right] \leq \sigma_{1} & \Longleftrightarrow \frac{-\mathbf{y}^{T} \boldsymbol{\mu}+\sqrt{\mathbf{y}^{T} \sum \mathbf{y}+\left(-\mathbf{y}^{T} \boldsymbol{\mu}\right)^{2}}}{2} \leq \sigma_{1} \\
& \Longleftrightarrow\left\{\begin{array}{l}
\mathbf{y}^{T} \Sigma \mathbf{y}-4 \sigma_{1} \mathbf{y}^{T} \boldsymbol{\mu} \leq 4 \sigma_{1}^{2}, \\
\mathbf{y}^{T} \boldsymbol{\mu} \geq-2 \sigma_{1} .
\end{array}\right.
\end{aligned}
$$

In fact, it can verify that

$$
\min _{\mathbf{y}}\left\{\mathbf{y}^{T} \boldsymbol{\mu}: \mathbf{y}^{T} \sum \mathbf{y}-4 \sigma_{1} \mathbf{y}^{T} \boldsymbol{\mu} \leq 4 \sigma_{1}^{2}\right\}>-2 \sigma_{1} .
$$

Hence, inequality constraint $\mathbf{y}^{T} \boldsymbol{\mu} \geq-2 \sigma_{1}$ is redundant. Thus, for any preset $\sigma_{1}>0$, we can rewrite problem (20) as

$$
\begin{gathered}
\mathrm{WCLPM}_{1}: \max _{\mathbf{y}}\left\{\mathbf{y}^{T} \boldsymbol{\mu}: \mathbf{y}^{T} \Sigma \mathbf{y}-4 \sigma_{1} \mathbf{y}^{T} \boldsymbol{\mu} \leq 4 \sigma_{1}^{2},\right. \\
\left.M^{T} \mathbf{y}=\mathbf{d}, \mathbf{e}^{T} \mathbf{y}=0\right\} .
\end{gathered}
$$

Here, we call $2 \sigma_{1}$ the upper bound of tracking error under $\mathrm{WCLPM}_{1}$ constraint and denote the upper bound of tracking error by $\mathrm{TE}_{1}=2 \sigma_{1}$. The following lemma gives the feasible condition of problem (33).

Lemma 9. If $\sigma_{1}$ is chosen to satisfy

$$
\sigma_{1}>\sigma_{1}^{*}
$$


then problem (33) is feasible, where

$$
\begin{aligned}
& \sigma_{1}^{*} \\
& =\frac{\sqrt{\left(a_{1} b_{0}-a_{0} b_{1}\right)^{2}+\left(a_{1} c_{1}-b_{1}^{2}+a_{1}\right)\left(a_{1} c_{0}+a_{0}^{2}\right)}-\left(a_{1} b_{0}-a_{0} b_{1}\right)}{2\left(a_{1} c_{1}-b_{1}^{2}+a_{1}\right)} .
\end{aligned}
$$

Proof. Let

$$
f(\mathbf{y})=\mathbf{y}^{T} \Sigma \mathbf{y}-4 \sigma_{1} \mathbf{y}^{T} \boldsymbol{\mu},
$$

and $\mathcal{S}_{11}=\left\{\mathbf{y}: f(\mathbf{y}) \leq 4 \sigma_{1}^{2}\right\}, \mathcal{S}_{12}=\left\{\mathbf{y} \in \mathbb{R}^{n}: M^{T} \mathbf{y}=\mathbf{d}, \mathbf{e}^{T} \mathbf{y}=\right.$ $0\}$. Then this lemma is to verify that the set $\mathcal{S}_{1}=\mathcal{S}_{11} \cap \mathcal{S}_{12}$ is nonempty for any $\sigma_{1}>\sigma_{1}^{*}$. Now, for any $\sigma_{1}>0$, consider the following subproblem:

$$
\min _{\mathbf{y}}\left\{f(\mathbf{y})=\mathbf{y}^{T} \Sigma \mathbf{y}-4 \sigma_{1} \mathbf{y}^{T} \boldsymbol{\mu}: M^{T} \mathbf{y}=\mathbf{d}, \mathbf{e}^{T} \mathbf{y}=0\right\},
$$

and denote its optimal value by $f_{\sigma_{1}}$ which is a quadratic function of parameter $\sigma_{1}$. Thus, (for the computation of $f_{\sigma_{1}}$ and $\sigma_{1}^{*}$, see Appendix for detail.),

$$
f_{\sigma_{1}} \leq 4 \sigma_{1}^{2}
$$

gives a quadratic inequality with respect to $\sigma_{1}$. Solving (38), we have that the equality in (38) holds when $\sigma_{1}=\sigma_{1}^{*}>0$, where $\sigma_{1}^{*}$ is given by (35). Hence, set $\mathcal{S}_{1}$ to be nonempty and has at least an element when $\sigma_{1}>\sigma_{1}^{*}$. This implies problem (33) is feasible. The proof is completed.

Lemma 9 indicates that problem $\mathrm{WCLPM}_{1}$ is well defined and meaningful. The following results give the explicit solution of problem WCLPM . $_{1}$

Theorem 10. Let the condition of Lemma 9 be satisfied. Then the optimal solution of problem (33) can be expressed explicitly as

$$
\begin{aligned}
\mathbf{y}_{1}^{*}= & \left(\lambda_{1}+2 \sigma_{1}\right)\left(\Sigma^{-1} B \boldsymbol{\mu}-\frac{b_{1}}{a_{1}} \Sigma^{-1} B \mathbf{e}\right) \\
& +a_{0}\left(\frac{C \mathbf{d}}{a_{0}}-\frac{\Sigma^{-1} B \mathbf{e}}{a_{1}}\right),
\end{aligned}
$$

where

$$
=\frac{\sqrt{4\left(a_{1} c_{1}-b_{1}^{2}+a_{1}\right) \sigma_{1}^{2}+4\left(a_{1} b_{0}-a_{0} b_{1}\right) \sigma_{1}-\left(a_{1} c_{0}+a_{0}^{2}\right)}}{\sqrt{a_{1} c_{1}-b_{1}^{2}}} .
$$

Proof. See Theorem 4 in [37]; see also Appendix for detail.
3.3. The Mean-WCLPM $M_{2}$. In this subsection, we consider mean-WCLPM $\mathrm{W}_{2}$ portfolio problem. In view of Lemma 2(c) and Lemma 4, the WCLPM ${ }_{2}$ constraint can be formulated as

$$
\left(\left(-\mathbf{y}^{T} \boldsymbol{\mu}\right)_{+}\right)^{2}+\mathbf{y}^{T} \Sigma \mathbf{y} \leq \sigma_{2} .
$$

Then we can write problem (7) with $m=2$ as

$$
\mathrm{WCLPM}_{2}: \max _{\mathbf{y}}\left\{\mathbf{y}^{T} \boldsymbol{\mu}:(41), M^{T} \mathbf{y}=\mathbf{d}, \mathbf{e}^{T} \mathbf{y}=0\right\} \text {. }
$$

If we add constraint $\mathbf{y}^{T} \boldsymbol{\mu} \geq 0$, then problem (42) is nothing but the classical $\mathrm{M}-\mathrm{V}$ model with weights constraints. On the other hand, if we add constraint $\mathbf{y}^{T} \boldsymbol{\mu}<0$, then constraint (41) is

$$
\left(\mathbf{y}^{T} \boldsymbol{\mu}\right)^{2}+\mathbf{y}^{T} \Sigma \mathbf{y}=\mathbf{y}^{T}\left(\Sigma+\boldsymbol{\mu} \boldsymbol{\mu}^{T}\right) \mathbf{y} \leq \sigma_{2}
$$

which is more conservative than the variance of portfolio for the same preset value $\sigma_{2}$ since $\left(\Sigma+\boldsymbol{\mu} \boldsymbol{\mu}^{T}\right)-\Sigma=\boldsymbol{\mu} \boldsymbol{\mu}^{T}$ is a positive semidefinite matrix. We call $\sqrt{\sigma_{2}}$, instead of $\sigma_{2}$, the upper bound of tracking error under $\mathrm{WCLPM}_{2}$ constraint and denote the upper bound by $\mathrm{TE}_{2}=\sqrt{\sigma_{2}}$. Similar to Lemma 9, we can also give the feasible condition of problem (42).

Lemma 11. Let

$$
\sigma_{2}>\frac{a_{1} c_{0}+a_{0}^{2}}{a_{1}}+\frac{\left(\left(a_{0} b_{1}-a_{1} b_{0}\right)_{+}\right)^{2}}{a_{1}^{2}+a_{1}\left(a_{1} c_{1}-b_{1}^{2}\right)} .
$$

Then problem (42) is feasible.

Proof. Let

$$
\sigma_{2}^{*}=\min _{\mathbf{y}}\left\{\left(\left(-\mathbf{y}^{T} \boldsymbol{\mu}\right)_{+}\right)^{2}+\mathbf{y}^{T} \Sigma \mathbf{y}: M^{T} \mathbf{y}=\mathbf{d}, \mathbf{e}^{T} \mathbf{y}=0\right\} .
$$

It follows from solving straightforwardly the problem that its optimal solution is

$$
\begin{aligned}
\widehat{\mathbf{y}}_{\sigma_{2}}^{*}= & \frac{\left[a_{1} v-\left(a_{1} b_{0}-a_{0} b_{1}\right)\right]}{a_{1} c_{1}-b_{1}^{2}}\left(\Sigma^{-1} B \boldsymbol{\mu}-\frac{b_{1}}{a_{1}} \Sigma^{-1} B \mathbf{e}\right) \\
& +a_{0}\left(\frac{C \mathbf{d}}{a_{0}}-\frac{\Sigma^{-1} B \mathbf{e}}{a_{1}}\right),
\end{aligned}
$$

where

$$
v=\frac{\left(a_{1} c_{1}-b_{1}^{2}\right)\left(a_{0} b_{1}-a_{1} b_{0}\right)_{+}}{a_{1}\left(a_{1}+a_{1} c_{1}-b_{1}^{2}\right)}+\frac{a_{1} b_{0}-a_{0} b_{1}}{a_{1}} .
$$

Then, we get that

$$
\sigma_{2}^{*}=\frac{a_{1} c_{0}+a_{0}^{2}}{a_{1}}+\frac{\left(\left(a_{0} b_{1}-a_{1} b_{0}\right)_{+}\right)^{2}}{a_{1}^{2}+a_{1}\left(a_{1} c_{1}-b_{1}^{2}\right)} .
$$

Hence, the problem (42) is feasible when $\sigma_{2}$ satisfies $\sigma_{2}>\sigma_{2}^{*}$. This completes the proof. 
By Lemma 11, problem (42) is well-defined and meaningful. The following results give the explicit expression of solution of problem (42).

Theorem 12. Let the condition of Lemma 11 be satisfied. Then problem (42) has the explicit solution as follows:

$$
\begin{aligned}
\mathbf{y}_{2}^{*}= & \frac{a_{1} v^{*}-\left(a_{1} b_{0}-a_{0} b_{1}\right)}{a_{1} c_{1}-b_{1}^{2}}\left[\Sigma^{-1} B \boldsymbol{\mu}-\frac{b_{1}}{a_{1}} \Sigma^{-1} B \mathbf{e}\right] \\
& +a_{0}\left[\frac{C \mathbf{d}}{a_{0}}-\frac{\Sigma^{-1} B \mathbf{e}}{a_{1}}\right],
\end{aligned}
$$

where

$$
v^{*}= \begin{cases}\frac{\sqrt{a_{1} a_{2}} \sqrt{\left(a_{1}+a_{2}\right)\left(a_{1} \sigma_{2}-c_{2}\right)-\left(\left(-b_{2}\right)_{+}\right)^{2}}}{a_{1}\left(a_{1}+a_{2}\right)} \\ +\frac{\sqrt{a_{2}\left(a_{1} \sigma_{2}-c_{2}\right)}\left(1-\sqrt{\frac{a_{1}}{a_{1}}}\right) \frac{\left(b_{2}\right)_{+}}{b_{2}}}{+\frac{\left(a_{1}+a_{2}\right) b_{2}+a_{2}\left(-b_{2}\right)_{+}}{a_{1}\left(a_{1}+a_{2}\right)},} \\ \frac{b_{2}}{a_{1}}+\frac{\sqrt{a_{2}\left(a_{1} \sigma_{2}-c_{2}\right)}}{a_{1}} \geq 0, & \sigma_{2}>\frac{b_{2}^{2}}{a_{1} a_{2}}+\frac{b_{2}}{a_{1} a_{2}}+\frac{c_{2}}{a_{1}} ;\end{cases}
$$

Proof. See Theorem 5 in [37]; see also Appendix for detail.

We mention that the optimal solution, $\mathbf{y}_{2}^{*}$, of problem (42) is consistent with problem (25) when $b_{2} \geq 0$ or $\sigma_{2}>b_{2}^{2} / a_{1} a_{2}+$ $c_{2} / a_{1}$. This is not surprising. Because the term $\left(\left(-\mathbf{y}^{T} \boldsymbol{\mu}\right)_{+}\right)^{2}$ vanishes in problem (42) in these cases.

\section{Numerical Results}

In this section, we consider the performance of models $\mathrm{WCLPM}_{1}$, WCLPM ${ }_{2}$, and MV using real market data. We choose ten stock indexes from Shanghai Stock Exchange (SHH) and Shenzhen Stock Exchange (SHZ), which are called domestic assets, and four stock indexes from other stock exchanges which are called foreign assets. All fourteen risky assets are listed in Table 1. Our data covers the period from January 1, 2000 to March 18, 2011 and includes 2663 samples for each asset. We group the whole period into two subperiods; that is, the first subperiod is from January 1, 2000 to April 21, 2006 and the second subperiod is from April 24, 2006 to March 18, 2011. The data in the first subperiod includes 1663 samples of each asset and is used as the insample test set. Other 1000 samples are used as the out-ofsample test set. The statistic properties of samples of all assets are reported in Table 2 . We take the benchmark as the naive $1 / N$ portfolio [38] with $N=14$. We fix TE $=0.05$ in the next numerical experiment.
TABLE 1: Chosen 14 indexes as the risky assets.

\begin{tabular}{lcc}
\hline Number & Asset name & Symbols \\
\hline & Domestic assets \\
1 & SSE Composite Index & $001 . S S$ \\
2 & SSE A-share Index & $002 . S S$ \\
3 & SSE B-share Index & $003 . S S$ \\
4 & SSE Industrial Index & $004 . S S$ \\
5 & SSE Commercial Index & $005 . S S$ \\
6 & SSE Properties Index & $006 . S S$ \\
7 & SSE Utilities Index & $007 . S S$ \\
8 & SSE Component Index & $001 . S Z$ \\
9 & SSE Component A & $002 . S Z$ \\
10 & SSE Composite Index & $106 . S Z$ \\
& Foreign Assets & \\
11 & BSE SENSEX & BSESN \\
12 & FTSE Bursa Malaysia KLCI & KLSE \\
13 & Hang Seng Index & HSI \\
14 & NIKKEI 225 & N225 \\
\hline
\end{tabular}

Here, we compare the performance of three models: $\mathrm{WCLPM}_{1}, \mathrm{MVMWC}$, and IRMWC (information ratio problem with multiple weights constraints, i.e., problem (24)). We consider the following three cases of weights constraints:

$$
\begin{gathered}
p=1: w_{11}+w_{12}+w_{13}+w_{14} \leq \frac{1}{20} . \\
p=2:\left\{\begin{array}{l}
w_{11}+w_{12}+w_{13}+w_{14} \leq \frac{1}{20} \\
w_{1}+w_{3}+w_{5}+w_{11}+w_{14} \leq \frac{1}{14}
\end{array}\right. \\
p=4:\left\{\begin{array}{l}
w_{11}+w_{12}+w_{13}+w_{14} \leq \frac{1}{20} \\
w_{1}+w_{3}+w_{5}+w_{11}+w_{14} \leq \frac{1}{14} \\
w_{7}+w_{12}+w_{14} \leq \frac{1}{14} \\
w_{8}+w_{2}+w_{13}+w_{14} \leq \frac{1}{20}
\end{array}\right.
\end{gathered}
$$

The wealth invested to foreign assets is restricted by the weights constraint. Table 3 gives the return, variance, and IR of optimal portfolios obtained by these models. Figures 1, 2, and 3 give the comparisons of portfolio value obtained in these models. The portfolio value in these figures is computed by

$$
\mathrm{PV}_{t}=\sum_{i=1}^{14} w_{i} \times \text { Asset }_{i t}
$$

where $\mathbf{w}=\left(w_{1}, \ldots, w_{14}\right)^{T}$ is the obtained optimal portfolios and Asset ${ }_{i}$ is the value of the $i$ th market index at the $t$ th exchange day. Some interesting results from our numerical comparisons can be found as follows.

(1) The tracking error of in-sample data from Figures 1-3 is less than that of out-of-sample data, and the tracking error of model IRMWC is the least among these models whatever 
TABLE 2: Asset market daily returns from January 1, 2000 to March 18, 2011.

\begin{tabular}{|c|c|c|c|c|}
\hline Asset & Mean $\left(10^{-3}\right)$ & Standard deviation & Minimum & Maximum \\
\hline \multicolumn{5}{|c|}{ Panel A: in-sample data: from January 01, 2000 to April 21, 2006} \\
\hline 001.SS & 0.4263 & 0.2019 & -0.0654 & 0.1096 \\
\hline 002.SS & 0.4194 & 0.1970 & -0.0651 & 0.1098 \\
\hline 003.SS & 0.8951 & 0.4687 & -0.1029 & 0.1840 \\
\hline 004.SS & 0.4381 & 0.1997 & -0.0631 & 0.1137 \\
\hline 005.SS & 0.3720 & 0.2390 & -0.0753 & 0.1039 \\
\hline 006.SS & 0.2563 & 0.3519 & -0.0974 & 0.1054 \\
\hline 007.SS & 0.5023 & 0.1960 & -0.0634 & 0.1089 \\
\hline 001.SZ & 0.4986 & 0.2282 & -0.0693 & 0.1163 \\
\hline 002.SZ & 0.4399 & 0.2284 & -0.0680 & 0.1208 \\
\hline 106.SZ & 0.2936 & 0.2172 & -0.0682 & 0.1056 \\
\hline BSESN & 0.5748 & 0.2476 & -0.1181 & 0.1046 \\
\hline KLSE & 0.2094 & 0.0862 & -0.0634 & 0.0649 \\
\hline HSI & 0.1150 & 0.1749 & -0.0929 & 0.0601 \\
\hline $\mathrm{N} 225$ & -0.0510 & 0.2112 & -0.0901 & 0.0722 \\
\hline \multicolumn{5}{|c|}{ Panel B: out-of-sample data: from April 24, 2006 to March 18, 2011} \\
\hline 001.SS & 0.0172 & 0.4691 & -0.1130 & 0.0903 \\
\hline 002.SS & 0.0138 & 0.4692 & -0.1128 & 0.0903 \\
\hline 003.SS & 0.6245 & 0.6482 & -0.1572 & 0.0937 \\
\hline 004.SS & 0.1737 & 0.4982 & -0.1186 & 0.0895 \\
\hline 005.SS & 0.5640 & 0.5404 & -0.1261 & 0.0918 \\
\hline 006.SS & 0.1804 & 0.9067 & -0.1817 & 0.0951 \\
\hline 007.SS & 0.1058 & 0.5745 & -0.1365 & 0.0938 \\
\hline 001.SZ & 0.4622 & 0.5780 & -0.1210 & 0.0916 \\
\hline 002.SZ & 0.4951 & 0.5776 & -0.1206 & 0.0916 \\
\hline 106.SZ & 0.6488 & 0.5608 & -0.1339 & 0.0852 \\
\hline BSESN & 0.2246 & 0.4266 & -0.1268 & 0.1599 \\
\hline KLSE & 0.2428 & 0.2111 & -0.1925 & 0.1986 \\
\hline HSI & 0.0759 & 0.4654 & -0.1358 & 0.1341 \\
\hline N225 & -0.6399 & 0.3917 & -0.1272 & 0.1323 \\
\hline
\end{tabular}

the in-sample and out-of-sample data is. But model WCLPM from Table 3 has the best expectation return among these considered models for all $p=1,2,4$, and in-sample and outof-sample data. This is understandable since model WCLPM has an excess MV region where it also exceeds the return of MVMWC. Moreover, from the view of portfolio value, WCLPM $_{1}$ can not only exceed benchmark, but can also get greater terminal wealth than that of models MVMWC and IRMWC.

(2) It seems to be not understandable that the tracking errors of all models from Figures 4, 5, and 6 increases as the number of restricted assets $p$ increases. But it is in fact not surprising, because the feasible sets of all models reduce as $p$ increases; this clearly leads to a larger error relative to the case of without restricted assets. Additionally, from Figures 4-6, we also find that models WCLPM 1 and MVMWC are more sensible than model IRMWC with respect to the number of restricted assets.

\section{Conclusions}

We considered a numerical extension of three active portfolio selection problems with the worst-case $m=0-, 1-$ and, 2-order lower partial moment risk and multiple weights constraints which are proposed in [37]. Using ten stocks from China market and four stocks from other market, we compared the numerical performance with VTE and " $1 / \mathrm{N}$ " strategy. Clearly, WCLPM $\mathrm{Wrom}_{1}$ the numerical results can obtain the better expectation excess return and information ratio than when the tracking error taken is small. Model MVMWC will be another good choice when a large tracking error is required and sell-shorting is forbidden. 
TABLE 3: The comparisons of return, variance, and information ratio of optimal solutions.

\begin{tabular}{|c|c|c|c|}
\hline Model & Mean return $\left(10^{-3}\right)$ & Standard deviation & Information ratio \\
\hline \multicolumn{4}{|c|}{ Panel A: in-sample data } \\
\hline Benchmark & 0.3850 & $1.1993 e-04$ & - \\
\hline VTE & 0.4304 & 0.0527 & 0.0782 \\
\hline \multicolumn{4}{|c|}{$p=1$} \\
\hline WCLPM $_{1}$ & 0.4522 & 0.0548 & 0.0818 \\
\hline MVMWC & 0.4256 & 0.0510 & 0.0759 \\
\hline IRMWC & 0.1300 & 0.0170 & 0.0781 \\
\hline \multicolumn{4}{|c|}{$p=2$} \\
\hline WCLPM $_{1}$ & 0.4412 & 0.0548 & 0.0813 \\
\hline MVMWC & 0.4204 & 0.0510 & 0.0755 \\
\hline IRMWC & 0.1365 & 0.0173 & 0.0786 \\
\hline \multicolumn{4}{|c|}{$p=4$} \\
\hline WCLPM $_{1}$ & 0.4526 & 0.0543 & 0.0666 \\
\hline MVMWC & 0.4278 & 0.0512 & 0.0626 \\
\hline IRMWC & 0.1325 & 0.0139 & 0.0607 \\
\hline \multicolumn{4}{|c|}{ Panel B: out-of-sample data } \\
\hline Benchmark & 0.2278 & $3.3696 e-004$ & - \\
\hline MV & 0.3925 & 0.0631 & 0.0102 \\
\hline \multicolumn{4}{|c|}{$p=1$} \\
\hline WCLPM $_{1}$ & 0.4282 & 0.0642 & 0.0104 \\
\hline MVMWC & 0.4033 & 0.0598 & 0.0099 \\
\hline IRMWC & 0.1237 & 0.0235 & 0.0103 \\
\hline \multicolumn{4}{|c|}{$p=2$} \\
\hline WCLPM $_{1}$ & 0.3851 & 0.0637 & 0.0102 \\
\hline MVMWC & 0.3542 & 0.0593 & 0.0101 \\
\hline IRMWC & 0.1032 & 0.0237 & 0.0102 \\
\hline \multicolumn{4}{|c|}{$p=4$} \\
\hline WCLPM $_{1}$ & 0.4268 & 0.0649 & 0.0101 \\
\hline MVMWC & 0.4006 & 0.0611 & 0.0101 \\
\hline IRMWC & 0.1260 & 0.0208 & 0.0101 \\
\hline
\end{tabular}

\section{Appendix}

\section{Technical Proofs}

Proof of Theorem 8. Let $\mathbf{y}_{0}^{*}$ be an optimal solution of problem (24). Then, $\mathbf{y}_{0}^{*}$ must satisfy the first order optimal condition of problem (24). Thus, we have

$$
\frac{\boldsymbol{\mu}}{\sqrt{\left(\mathbf{y}_{0}^{*}\right)^{T} \Sigma \mathbf{y}_{0}^{*}}}-\frac{\left(\boldsymbol{\mu}^{T} \mathbf{y}_{0}^{*}\right)}{\sqrt{\left(\mathbf{y}_{0}^{*}\right)^{T} \Sigma \mathbf{y}_{0}^{*}}} \frac{\Sigma \mathbf{y}_{0}^{*}}{\left(\mathbf{y}_{0}^{*}\right)^{T} \Sigma \mathbf{y}_{0}^{*}}-\lambda_{01} \mathbf{e}-M \lambda_{02}=0
$$

where $\lambda_{01} \in \mathbb{R}, \lambda_{02} \in \mathbb{R}^{p}$ are the Lagrange multipliers. Let $\hat{\lambda}_{01}=\lambda_{01} \sqrt{\left(\mathbf{y}_{0}^{*}\right)^{T} \Sigma \mathbf{y}_{0}^{*}}$ and $\hat{\boldsymbol{\lambda}}_{02}=\lambda_{02} \sqrt{\left(\mathbf{y}_{0}^{*}\right)^{T} \Sigma y_{0}^{*}}$; then equality (A.1) can be reduced as

$$
\boldsymbol{\mu}-\tau_{0} \Sigma \mathbf{y}_{0}^{*}-\widehat{\lambda}_{01} \mathbf{e}-M \widehat{\lambda}_{02}=0,
$$

where $\tau_{0}=\left(\boldsymbol{\mu}^{T} \mathbf{y}_{0}^{*}\right) /\left(\mathbf{y}_{0}^{*}\right)^{T} \Sigma \mathbf{y}_{0}^{*}$ is a constant. Thus, combining (A.2) and the equality constraints $M^{T} \mathbf{y}_{0}^{*}=\mathbf{d}$ and $\mathbf{e}^{T} \mathbf{y}_{0}^{*}=0$, we get that

$$
\mathbf{y}_{\tau_{0}}^{*}=\frac{b_{1}}{\tau_{0}}\left(\frac{\Sigma^{-1} B \boldsymbol{\mu}}{b_{1}}-\frac{\Sigma^{-1} B \mathbf{e}}{a_{1}}\right)+a_{0}\left(\frac{C \mathbf{d}}{a_{0}}-\frac{\Sigma^{-1} B \mathbf{e}}{a_{1}}\right) .
$$

This completes the proof.

The Computation of $f_{\sigma_{1}}$ and $\sigma_{1}^{*}$. Consider the following subproblem:

$$
\min _{\mathbf{y}}\left\{f(\mathbf{y})=\mathbf{y}^{T} \Sigma \mathbf{y}-4 \sigma_{1} \mathbf{y}^{T} \boldsymbol{\mu} \mid \text { s.t. } M^{T} \mathbf{y}=\mathbf{d}, \mathbf{e}^{T} \mathbf{y}=0\right\},
$$

and denote its optimal value by $f_{\sigma_{1}}$. Let $\mathbf{y}_{\sigma_{1}}^{*}$ be its optimal solution. Then, by the KKT optimal condition, we have

$$
\begin{gathered}
2 \Sigma \mathbf{y}_{\sigma_{1}}^{*}-4 \sigma_{1} \boldsymbol{\mu}+\lambda_{11} \mathbf{e}+M \boldsymbol{\lambda}_{12}=0, \\
M^{T} \mathbf{y}_{\sigma_{1}}^{*}=\mathbf{d}, \quad \mathbf{e}^{T} \mathbf{y}_{\sigma_{1}}^{*}=0,
\end{gathered}
$$




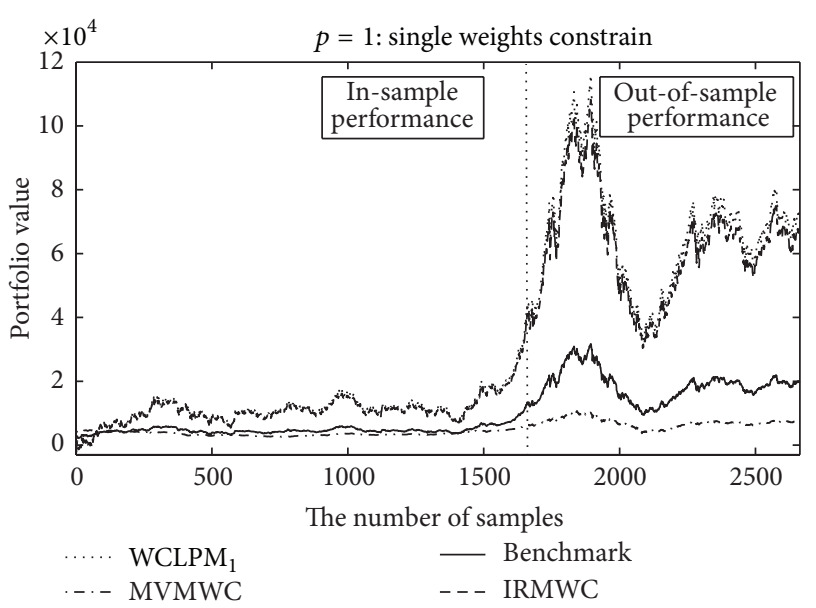

FIGURE 1: The comparison of portfolio values of models $\mathrm{WCLPM}_{1}$, MVMWC, IRMWC, and benchmark for in-sample and out-ofsample data when there is single weight constraint.

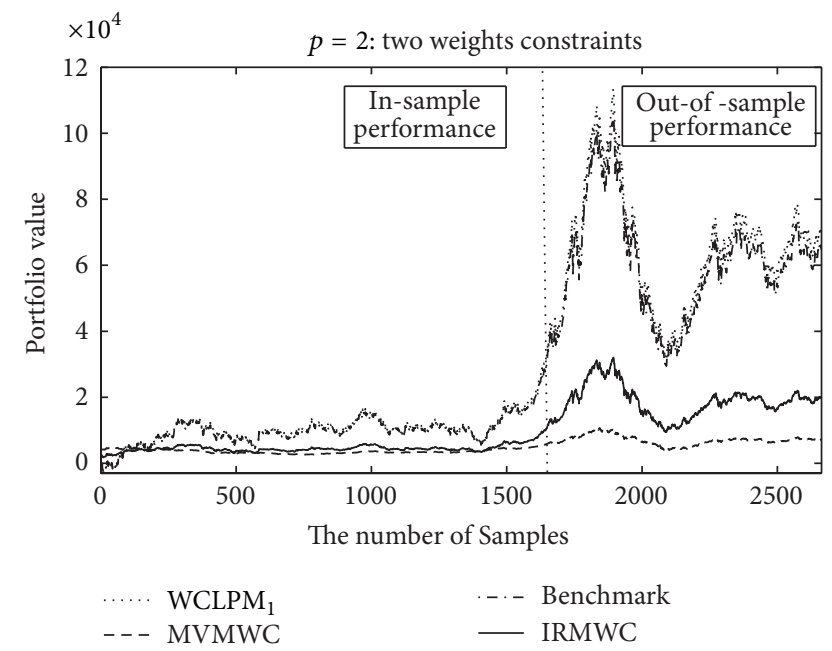

FIGURE 2: The comparison of portfolio values of models $\mathrm{WCLPM}_{1}$, MVMWC, IRMWC, and benchmark for in-sample and out-ofsample data when there are two weights constraints.

where $\lambda_{11} \in \mathbb{R}$ and $\lambda_{12} \in \mathbb{R}^{p}$ are the Lagrange multiplier. Solving the equality system of KKT conditions, we get

$$
\mathbf{y}_{\sigma_{1}}^{*}=2 \sigma_{1}\left(\Sigma^{-1} B \boldsymbol{\mu}-\frac{b_{1}}{a_{1}} \Sigma^{-1} B \mathbf{e}\right)+a_{0}\left(\frac{C \mathbf{d}}{a_{0}}-\frac{\Sigma^{-1} B \mathbf{e}}{a_{1}}\right) .
$$

Substituting it into the objective function and using some results in Lemma 5 , it follows that

$$
\begin{aligned}
f_{\sigma_{1}}= & -\frac{4\left(a_{1} c_{1}-b_{1}^{2}\right)}{a_{1}} \sigma_{1}^{2}-\frac{4\left(a_{1} b_{0}-a_{0} b_{1}\right)}{a_{1}} \sigma_{1} \\
& +\frac{a_{1} c_{0}+a_{0}^{2}}{a_{1}}
\end{aligned}
$$

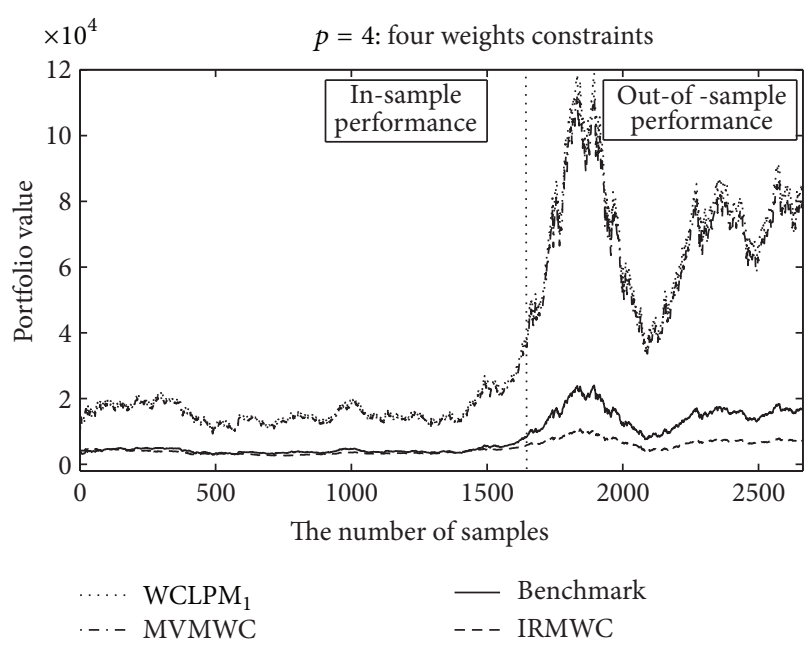

FIGURE 3: The comparison of portfolio values of models WCLPM $_{1}$, MVMWC, IRMWC, and benchmark for in-sample and out-ofsample data when there are four weights constraints.

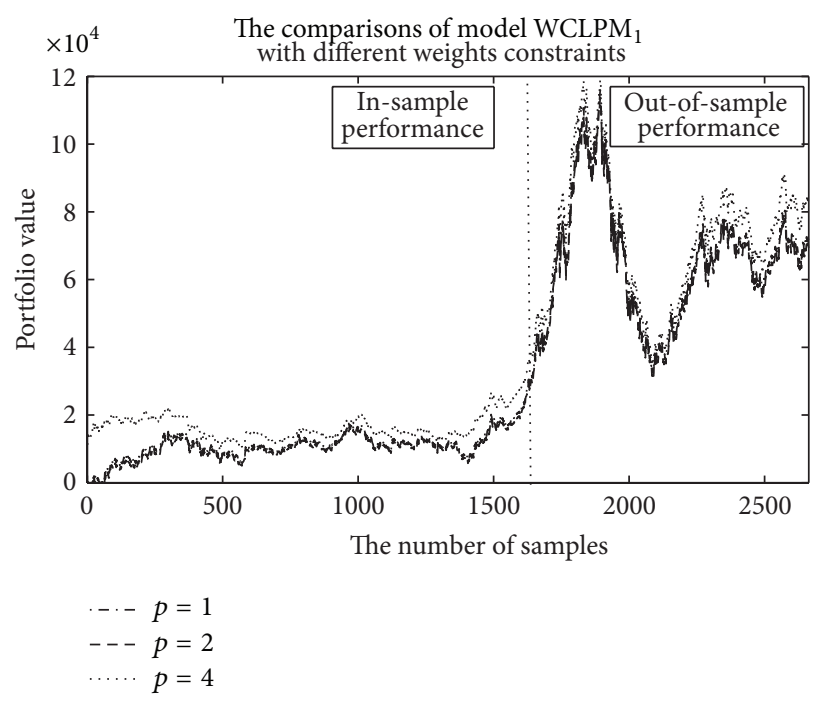

FIGURE 4: The comparison of portfolio values of model WCLPM for in-sample and out-of-sample data and different values of $p$.

and it is a quadratic function of parameter $\sigma_{1}$. Solving directly the quadratic equation in $\sigma_{1}$

$$
f_{\sigma_{1}}=4 \sigma_{1}^{2},
$$

we get a positive root of this equation as

$$
\begin{aligned}
& \sigma_{1}^{*}=( \sqrt{\left(a_{1} b_{0}-a_{0} b_{1}\right)^{2}+\left(a_{1} c_{1}-b_{1}^{2}+a_{1}\right)\left(a_{1} c_{0}+a_{0}^{2}\right)} \\
&\left.-\left(a_{1} b_{0}-a_{0} b_{1}\right)\right) \times\left(2\left(a_{1} c_{1}-b_{1}^{2}+a_{1}\right)\right)^{-1} .
\end{aligned}
$$

This obtains equality (35). Thus, inequality $f_{\sigma_{1}}<4 \sigma_{1}^{2}$ holds when $\sigma_{1}>\sigma_{1}^{*}$. This is the conclusion of Lemma 9 . 


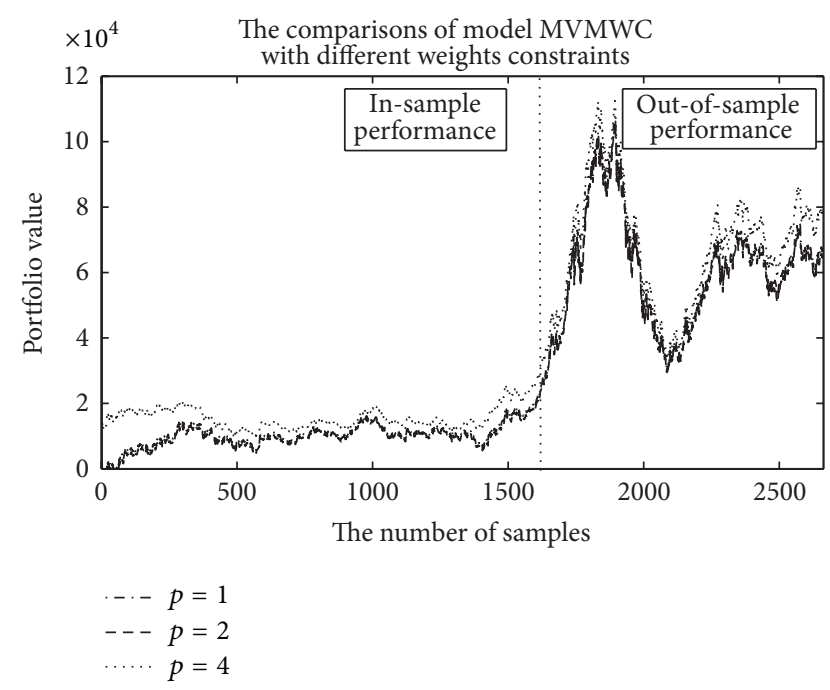

Figure 5: The comparison of portfolio values of model MVMWC, for in-sample and out-of-sample data and different values of $p$.

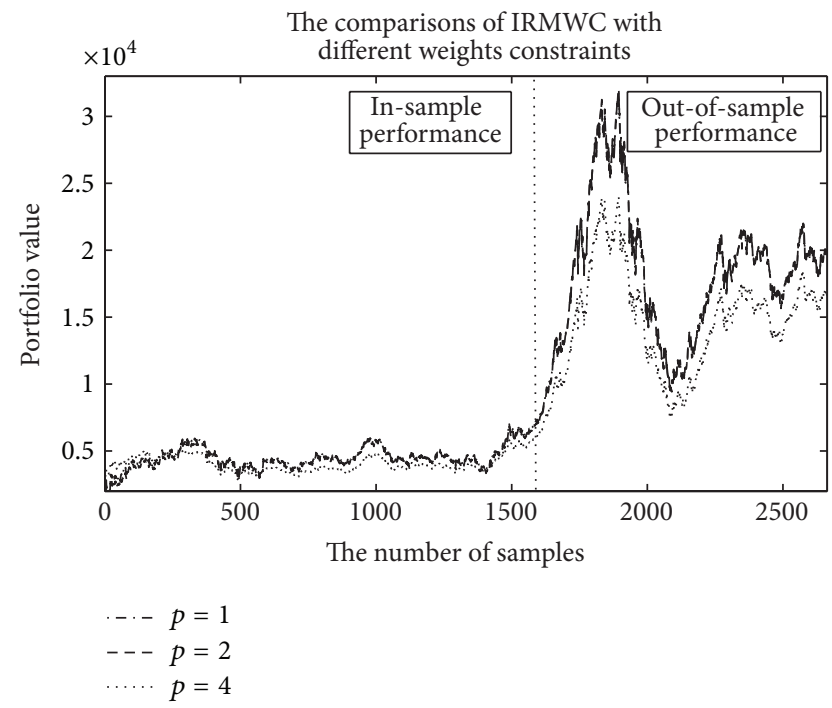

FIGURE 6: The comparison of portfolio values of model IRMWC for in-sample and out-of-sample data and different values of $p$.

Proof of Theorem 10. Let $\mathbf{y}_{1}^{*}$ be the optimal solution of problem (33). Then from KKT condition, we have

$$
\begin{gathered}
\left(1+4 \sigma_{1} \lambda_{1}\right) \boldsymbol{\mu}-2 \lambda_{11} \Sigma \mathbf{y}_{1}^{*}-\lambda_{12} \mathbf{e}-M \lambda_{13}=0 \\
\left(\mathbf{y}_{1}^{*}\right)^{T} \Sigma \mathbf{y}_{1}^{*}-4 \sigma_{1} \mathbf{y}_{1}^{*} \boldsymbol{\mu}=4 \sigma_{1}^{2} \\
M \mathbf{y}_{1}^{*}=\mathbf{d} \\
\mathbf{e}^{T} \mathbf{y}_{1}^{*}=0
\end{gathered}
$$

where $\lambda_{11} \geq 0, \lambda_{12} \in \mathbb{R}$, and $\lambda_{13} \in \mathbb{R}^{p}$ are the Lagrange multipliers. Solving directly KKT system (A.10), it gets the conclusions of Theorem 10. The proof is finished.
Proof of Theorem 12. Let $v=\mathbf{y}^{T} \boldsymbol{\mu}$. Then we can rewrite problem (42) as

$$
\begin{array}{cl}
\max _{\mathbf{y}, v} & \boldsymbol{\mu}^{T} \mathbf{y} \\
\text { s.t. } & \left((-v)_{+}\right)^{2}+\mathbf{y}^{T} \sum \mathbf{y} \leq \sigma_{2} \\
& M^{T} \mathbf{y}=\mathbf{d} \\
& \mathbf{e}^{T} \mathbf{y}=0 \\
& \boldsymbol{\mu}^{T} \mathbf{y}=v
\end{array}
$$

here, we view $v$ as an auxiliary variable. The Lagrange function of this problem is

$$
\begin{aligned}
& L\left(\mathbf{y}, v ; \lambda_{21}, \lambda_{22}, \lambda_{23}\right) \\
& =\boldsymbol{\mu}^{T} \mathbf{y}-\lambda_{21} \mathbf{e}^{T} \mathbf{y}-\lambda_{22}^{T}\left(M^{T} \mathbf{y}-\widehat{\mathbf{d}}\right) \\
& \quad-\lambda_{23}\left[\left((-v)_{+}\right)^{2}+\mathbf{y}^{T} \Sigma \mathbf{y}-\sigma_{2}\right]-\lambda_{24}\left(\boldsymbol{\mu}^{T} \mathbf{y}-v\right),
\end{aligned}
$$

where $\lambda_{21} \in \mathbb{R}, \lambda_{22} \in \mathbb{R}^{p}, \lambda_{23} \geq 0$, and $\lambda_{24} \in \mathbb{R}$ are the Lagrange multipliers. Let the pair $\left(\mathbf{y}_{2}^{*}, v^{*}\right)$ be the optimal solution of (A.11). Then the pair $\left(\mathbf{y}_{2}^{*}, v^{*}\right)$ satisfies the first optimal condition; that is,

$$
\begin{gathered}
\frac{\partial L}{\partial \mathbf{y}}=-2 \lambda_{23} \Sigma \mathbf{y}_{2}^{*}-\lambda_{21} \mathbf{e}-M \lambda_{22}+\left(1-\lambda_{24}\right) \boldsymbol{\mu}=0 \\
\frac{\partial L}{\partial v}=-2 \lambda_{23}\left(-v^{*}\right)_{+}+\lambda_{24}=0 \\
\left(\left(-v^{*}\right)_{+}\right)^{2}+\left(\mathbf{y}_{2}^{*}\right)^{T} \Sigma \mathbf{y}_{2}^{*}=\sigma_{2} \\
M^{T} \mathbf{y}_{2}^{*}=\mathbf{d}, \quad \mathbf{e}^{T} \mathbf{y}_{2}^{*}=0, \quad \boldsymbol{\mu}^{T} \mathbf{y}_{2}^{*}=v^{*}
\end{gathered}
$$

By the first equation of (A.13), it gets that

$$
\mathbf{y}_{2}^{*}=-\frac{1}{2 \lambda_{23}}\left[\lambda_{21} \Sigma^{-1} \mathbf{e}+\Sigma^{-1} M \lambda_{22}-\left(1-\lambda_{24}\right) \Sigma^{-1} \boldsymbol{\mu}\right]
$$

Substituting $\mathbf{y}_{2}^{*}$ into equation $M^{T} \mathbf{y}_{2}^{*}=\mathbf{d}$, we obtain that

$\lambda_{22}$

$$
=-\left[\lambda_{21} A^{-1} M^{T} \Sigma^{-1} \mathbf{e}+2 \lambda_{23} A^{-1} \mathbf{d}-\left(1-\lambda_{24}\right) A^{-1} M^{T} \Sigma^{-1} \boldsymbol{\mu}\right],
$$

where $A=M^{T} \Sigma^{-1} M$. Substituting $\lambda_{22}$ into $\mathbf{y}_{2}^{*}$ and using Lemma 5, we further get

$$
\mathbf{y}_{2}^{*}=\frac{1}{2 \lambda_{23}}\left[\left(1-\lambda_{24}\right) \Sigma^{-1} B \boldsymbol{\mu}-\lambda_{21} \Sigma^{-1} B \mathbf{e}+2 \lambda_{23} C \mathbf{d}\right] \text {. }
$$

Combining equation $\mathbf{e}^{T} \mathbf{y}_{2}^{*}=0$, it gets that

$$
\lambda_{21}=\left(1-\lambda_{24}\right) \frac{b_{1}}{a_{1}}+\frac{2 a_{0}}{a_{1}} \lambda_{23} .
$$


Notice that $1-\lambda_{24}=1-2 \lambda_{23}\left(-v^{*}\right)_{+}$. Thus, $\mathbf{y}_{2}^{*}$ can be reduced as

$$
\begin{aligned}
\mathbf{y}_{2}^{*}= & \left(\frac{1}{2 \lambda_{23}}-\left(-v^{*}\right)_{+}\right)\left[\Sigma^{-1} B \boldsymbol{\mu}-\frac{b_{1}}{a_{1}} \Sigma^{-1} B \boldsymbol{\mu}\right] \\
& +a_{0}\left[\frac{C \mathbf{d}}{a_{0}}-\frac{\Sigma^{-1} B \mathbf{e}}{a_{1}}\right] .
\end{aligned}
$$

Substituting $\mathbf{y}_{2}^{*}$ into the last equation $\boldsymbol{\mu}^{T} \mathbf{y}_{2}^{*}=v^{*}$ of (A.13), we get

$$
\frac{1}{2 \lambda_{23}}=\frac{a_{1} v^{*}-\left(a_{1} b_{0}-a_{0} b_{1}\right)}{a_{1} c_{1}-b_{1}^{2}}+\left(-v^{*}\right)_{+} .
$$

On the other hand, substituting $\mathbf{y}_{2}^{*}$ into the third equation of (A.13), we can obtain another expression of $1 / 2 \lambda_{23}$ as

$$
\begin{aligned}
& \frac{1}{2 \lambda_{23}} \\
& =\frac{\sqrt{\left(a_{1} c_{1}-b_{1}^{2}\right)\left[a_{1} \sigma_{2}-\left(a_{1} c_{0}+a_{0}^{2}\right)-a_{1}\left(\left(-v^{*}\right)_{+}\right)^{2}\right]}}{a_{1} c_{1}-b_{1}^{2}} \\
& +\left(-v^{*}\right)_{+} .
\end{aligned}
$$

Hence, combining (A.19) and (A.20) and noticing the notations in Lemma 5, we have that $v^{*}$ satisfies the quadratic equation

$$
\begin{gathered}
a_{1}^{2}\left(v^{*}\right)^{2}+a_{1} a_{2}\left(\left(-v^{*}\right)_{+}\right)^{2}-2 a_{1} b_{2} v^{*}+b_{2}^{2} \\
-a_{2}\left(a_{1} \sigma_{2}-c_{2}\right)=0 .
\end{gathered}
$$

Next, we discuss the solutions of (A.21) by the different sign of $b_{2}$.

(1) $b_{2} \geq 0$. Notice that $v^{*}=\boldsymbol{\mu}^{T} \mathbf{y}_{2}^{*}$ is the expectation excess return of self-finance portfolio $\mathbf{y}_{2}^{*}$. Thus, generally speaking, $v^{*}$ is increasing as the preset tracking error $\sigma_{2}$ increases. Thus, (A.21) has only an efficient positive solution as follows. (Equation (A.21) has in this case another solution

$$
v^{*}=\frac{b_{2}}{a_{1}+a_{2}}-\frac{\sqrt{a_{1} a_{2}} \sqrt{\left(a_{1}+a_{2}\right)\left(a_{1} \sigma_{2}-c_{2}\right)}}{a_{1}\left(a_{1}+a_{2}\right)}<0,
$$

when $v^{*}<0$ and $\sigma_{2}$ is taken to satisfy $\sigma_{2}>\left(b_{2}^{2} / a_{1} a_{2}\right)+\left(c_{2} / a_{1}\right)$, but it is not an efficient solution since $v^{*}$ is decreasing as $\sigma_{2}$ increases)

$$
v^{*}=\frac{b_{2}}{a_{1}}+\frac{\sqrt{a_{2}\left(a_{1} \sigma_{2}-c_{2}\right)}}{a_{1}} \geq 0 .
$$

(2) $b_{2}<0$. If $v^{*} \leq 0$, then (A.21) has the solution

$$
v^{*}=\frac{b_{2}}{a_{1}+a_{2}}+\frac{\sqrt{a_{1} a_{2}} \sqrt{\left(a_{1}+a_{2}\right)\left(a_{1} \sigma_{2}-c_{2}\right)-b_{2}^{2}}}{a_{1}\left(a_{1}+a_{2}\right)} \leq 0,
$$

when $\sigma_{2}$ is chosen to satisfy $\sigma_{2}^{*} \leq \sigma_{2} \leq\left(b_{2}^{2} / a_{1} a_{2}\right)+\left(c_{2} / a_{1}\right)$. If $\sigma_{2}>\left(b_{2}^{2} / a_{1} a_{2}\right)+\left(c_{2} / a_{1}\right)$, then the side of right hand of equality (A.24) is positive and therefore equality (A.24) is not a solution of (A.21). In the case of $\sigma_{2}>\left(b_{2}^{2} / a_{1} a_{2}\right)+\left(c_{2} / a_{1}\right)$, the solution equation (A.21) has still the form of (A.23).

Summarizing the two cases above, we get that the solution of (A.21) can be expressed as (50). This completes the proof.

\section{Conflict of Interests}

The authors declare that there is no conflict of interests regarding the publication of this paper.

\section{Acknowledgments}

The authors are in debt to two anonymous referees and Lead Guest Editor, Professor Chuangxia Huang, for their constructive comments and suggestions to improve the quality of this paper. This work is supported by National Natural Science Foundations of China (nos. 71001045 and 71371090), Science Foundation of Ministry of Education of China (13YJCZH160), Natural Science Foundation of Jiangxi Province of China (20114BAB211008), and Jiangxi University of Finance and Economics Support Program Funds for Outstanding Youths.

\section{References}

[1] H. M. Markowitz, "Portfolio selection," Journal of Finance, vol. 7, pp. 77-91, 1952.

[2] H. Markowitz, "The optimization of a quadratic function subject to linear constraints," Naval Research Logistics Quarterly, vol. 3, pp. 111-133, 1956.

[3] R. Roll, "A mean-variance analysis of the tracking error," Journal of Portfolio Management, vol. 18, no. 1, pp. 13-22, 1992.

[4] G. J. Alexander and A. M. Baptista, "Active portfolio management with benchmarking: adding a value-at-risk constraint," Journal of Economic Dynamics \& Control, vol. 32, no. 3, pp. 779820, 2008.

[5] P. Jorion, "Portfolio optimization with tracking-error constraints," Financial Analysts Journal, vol. 59, no. 5, pp. 70-82, 2003.

[6] M. Rudolf, H. J. Wolter, and H. Zimmermann, "A linear model for tracking error minimization," Journal of Banking and Finance, vol. 23, no. 1, pp. 85-103, 1999.

[7] F. Wen and Z. Liu, "A copula-based correlation measure and its application in chinese stock market," International Journal of Information Technology and Decision Making, vol. 8, no. 4, pp. 787-801, 2009.

[8] F. Wen and X. Yang, "Skewness of return distribution and coefficient of risk premium," Journal of Systems Science \& Complexity, vol. 22, no. 3, pp. 360-371, 2009.

[9] Y. Zhao, "A dynamic model of active portfolio management with benchmark orientation," Journal of Banking and Finance, vol. 31, no. 11, pp. 3336-3356, 2007.

[10] H. H. Chena, H. T. Tsaib, and D. K. J. Linc, "Optimal meanvariance portfolio selection using Cauchy-Schwarz maximization," Applied economics, vol. 43, pp. 2795-2801, 2011. 
[11] P. Glabadanidis, "Robust and efficient strategies to track and outperform a benchmark," working paper, 2010, http://papers.ssrn.com/sol3/papers.cfm?abstract-id=1571250 .

[12] I. Guedj and J. Huang, "Are ETFs replacing index mutual funds?" Manuscript, 2009, http://papers.ssrn.com/sol3/ papers.cfm?abstract-id=1108728.

[13] V. Glode, "Why mutual funds 'Underperform"' Manuscript, 2010, http://papers.ssrn.com/sol3/papers.cfm?abstract_id=112 1436.

[14] C. Huang, C. Peng, X. Chen, and F. Wen, "Dynamics analysis of a class of delayed economic model," Abstract and Applied Analysis, vol. 2013, Article ID 962738, 12 pages, 2013.

[15] C. X. Huang, X. Gong, X. H. Chen, and F. H. Wen, "Measuring and forecasting volatility in Chinese stock market using HAR CJM model," Abstract and Applied Analysis, vol. 2013, Article ID 143194, 13 pages, 2013.

[16] S. Liu and R. Xu, "The effects of risk aversionon optimization," MSCI Barra Research, 2010, http://ssrn.com/abstract=1601412.

[17] M. Wang, C. Xu, F. Xu, and H. Xue, "A mixed 0-1 LP for index tracking problem with CVaR risk constraints," Annals of Operations Research, vol. 196, pp. 591-609, 2012.

[18] F. Wen and Z. Dai, "Modified Yabe-Takano nonlinear conjugate gradient method," Pacific Journal of Optimization, vol. 8, no. 2, pp. 347-360, 2012.

[19] H. M. Markowitz, Porfolio Selection: Ecient Diversification of Investment, John Willey, New York, NY, USA, 1959.

[20] V. S. Bawa, "Optimal rules for ordering uncertain prospects," Journal of Financial Economics, vol. 2, no. 1, pp. 95-121, 1975.

[21] V. S. Bawa and E. B. Lindenberg, "Capital market equilibrium in a mean-lower partial moment framework," Journal of Financial Economics, vol. 5, no. 2, pp. 189-200, 1977.

[22] P. C. Fishburn, "Mean-risk analysis with risk associated with below-target returns," American Economics Review, vol. 67, no. 2, pp. 116-126, 1977.

[23] L. Chen, S. He, and S. Zhang, "Tight bounds for some risk measures, with applications to robust portfolio selection," Operations Research, vol. 59, no. 4, pp. 847-865, 2011.

[24] H. Grootveld and W. Hallerbach, "Variance vs downside risk: is there really that much difference?" European Journal of Operational Research, vol. 114, no. 2, pp. 304-319, 1999.

[25] W. V. Harlow, "Asset allocation in a downside-risk framework," Financial Analysis Journal, vol. 475, pp. 28-40, 1991.

[26] F. Wen, L. Zhong, c. Xie, and S. David, "Study on the fractal and chaotic features of the Shanghai composite index," Fractals, vol. 20, no. 2, pp. 133-140, 2012.

[27] L. Yu, S. Zhang, and X. Y. Zhou, "A downside risk analysis based on financial index tracking models," in Stochastic Finance, part 1, pp. 213-236, 2006.

[28] S. Zhu, D. Li, and S. Wang, "Robust portfolio selection under downside risk measures," Quantitative Finance, vol. 9, no. 7, pp. 869-885, 2009.

[29] A. Ben-Tal and A. Nemirovski, "Robust convex optimization," Mathematics of Operations Research, vol. 23, no. 4, pp. 769-805, 1998.

[30] O. L. V. Costa and A. C. Paiva, "Robust portfolio selection using linear-matrix inequalities," Journal of Economic Dynamics \& Control, vol. 26, no. 6, pp. 889-909, 2002.

[31] L. El Ghaoui, M. Oks, and F. Oustry, "Worst-case value-atrisk and robust portfolio optimization: a conic programming approach," Operations Research, vol. 51, no. 4, pp. 543-556, 2003.
[32] D. Goldfarb and G. Iyengar, "Robust portfolio selection problems," Mathematics of Operations Research, vol. 28, no. 1, pp. 138, 2003.

[33] E. Erdoğan, D. Goldfarb, and G. Iyengar, "Robust active portfolio management," The Journal of Computational Finance, vol. 11, no. 4, pp. 71-98, 2008.

[34] A. F. Ling and C. X. Xu, "Robust portfolio selection involving options under a "marginal + joint" ellipsoidal uncertainty set," Journal of Computational and Applied Mathematics, vol. 236, no. 14, pp. 3373-3393, 2012.

[35] F. J. Fabozzi, D. Huang, and G. Zhou, "Robust portfolios: contributions from operations research and finance," Annals of Operations Research, vol. 176, pp. 191-220, 2010.

[36] I. Bajeux-Besnainou, R. Belhaj, D. Maillard, and R. Portait, "Portfolio optimization under tracking error and weights constraints," Journal of Financial Research, vol. 34, no. 2, pp. 295330, 2011.

[37] A. Ling, X. Yang, and L. Tang, "Robust LPM active portfolio selection problems with multiple weights constraints," Journal of Management Science in China, vol. 16, no. 8, pp. 32-46, 2013.

[38] V. DeMiguel, L. Garlappi, and R. Uppal, "Optimal versus naive diversification: how inefficient is the $1 / N$ portfolio strategy?" Review of Financial Studies, vol. 22, no. 5, pp. 1915-1953, 2009.

[39] D. Bertsimas and I. Popescu, "On the relation between option and stock prices: a convex optimization approach," Operations Research, vol. 50, no. 2, pp. 358-374, 2002.

[40] I. Popescu, "Robust mean-covariance solutions for stochastic optimization," Operations Research, vol. 55, no. 1, pp. 98-112, 2007. 


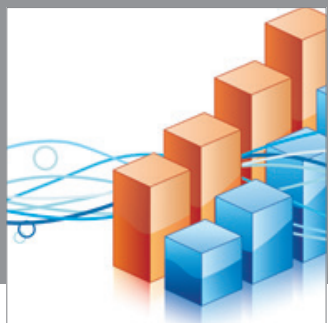

Advances in

Operations Research

mansans

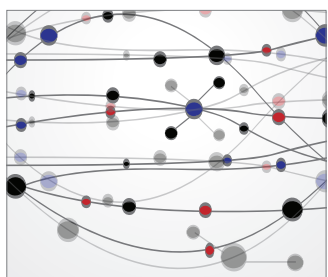

The Scientific World Journal
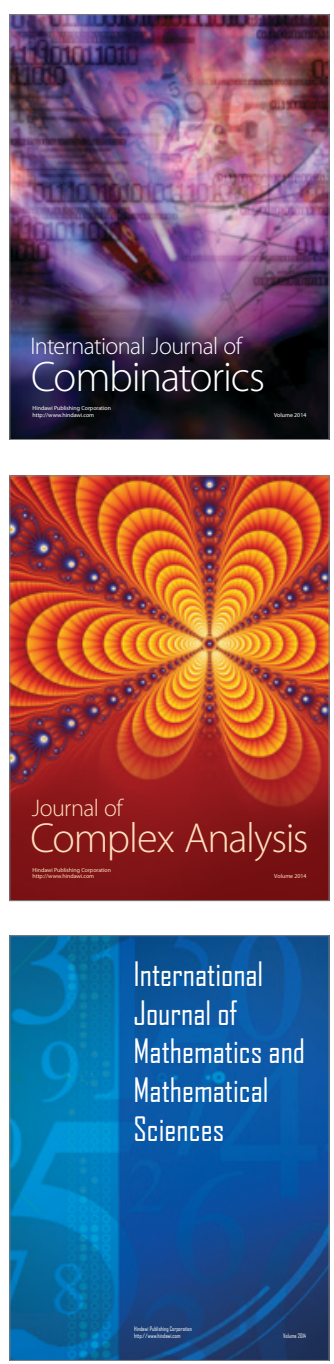
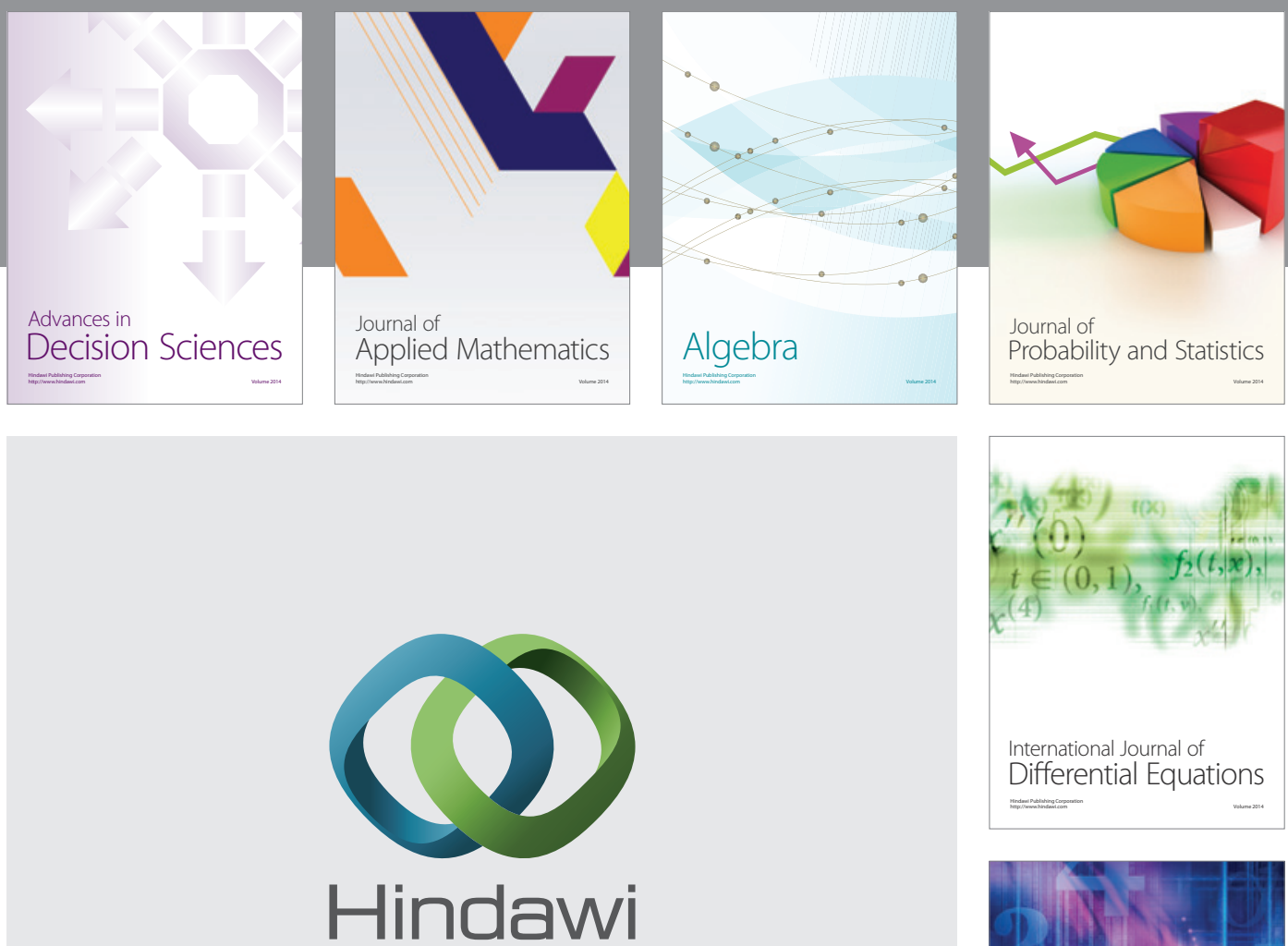

Submit your manuscripts at http://www.hindawi.com
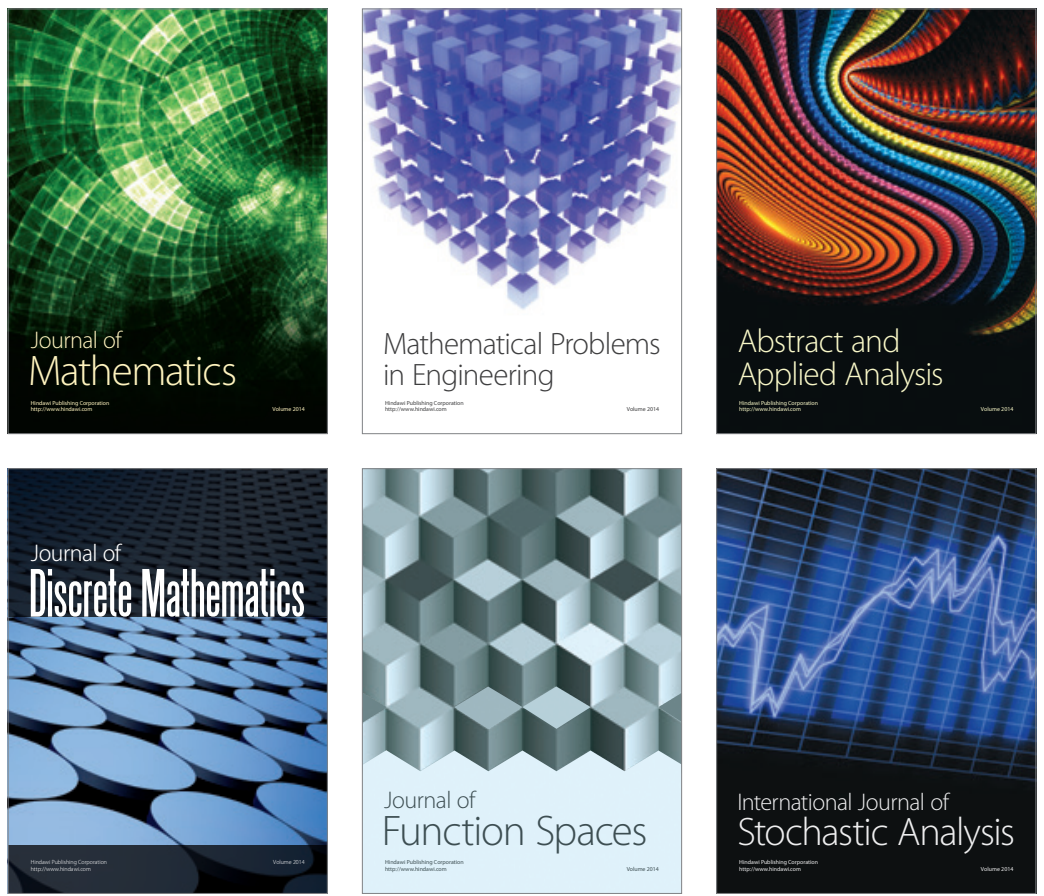

Journal of

Function Spaces

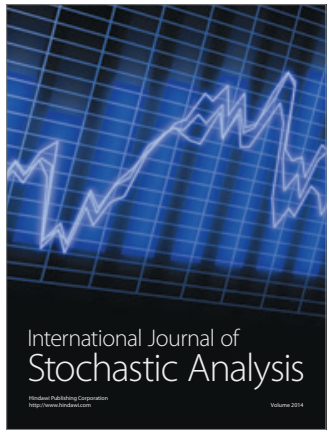

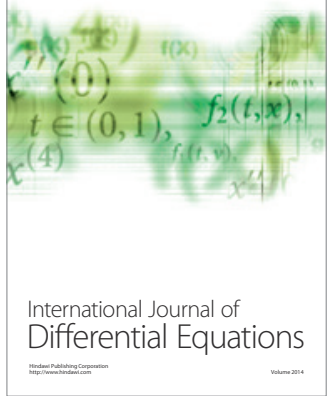
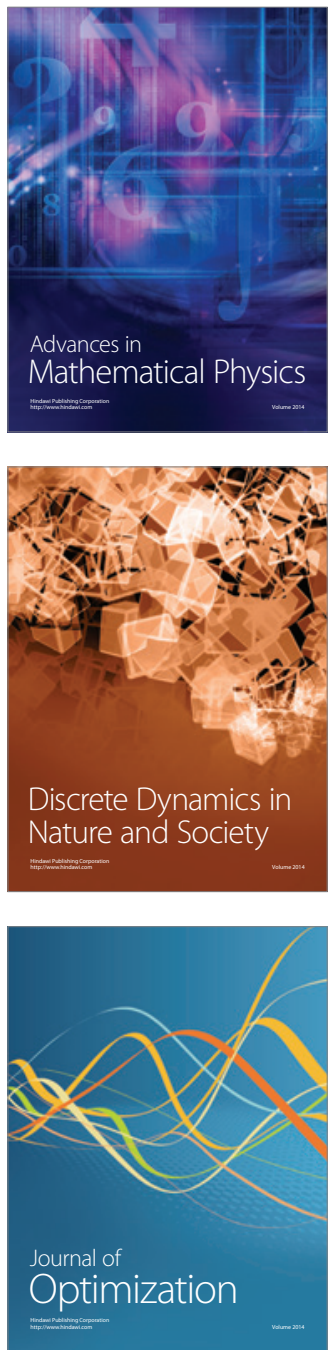